\title{
Shallow defect states in two-dimensional photonic crystals
}

\author{
K. B. Dossou ${ }^{1 *}$, L. C. Botten ${ }^{1}$, R. C. McPhedran ${ }^{2}$, C. G. Poulton ${ }^{1}$, A. A. Asatryan ${ }^{1}$ and C. Martijn de Sterke ${ }^{2}$ \\ ${ }^{1}$ CUDOS, Department of Mathematical Sciences, \\ University of Technology, Sydney, NSW 2007, Australia* and \\ ${ }^{2}$ CUDOS, School of Physics, University of Sydney, NSW 2006, Australia
}

(Dated: June 20, 2008)

\begin{abstract}
We investigate localized defect states near the edge of a band-gap in a two-dimensional photonic crystal. An asymptotic approach based on Green's functions leads to analytical results both for the frequency and for the spatial behaviour of the defect states. In particular, we find a simple exponential law which relates the change in frequency of the defect states to the relative change in electrical energy of the Bloch modes on the band-edge, and to the density of states in the photonic crystal. We find that the symmetries of the Bloch modes at band extrema play an important role in the manifestation and evolution of defect states. We confirm the analysis with numerical simulations based on the Fictitious Source Superposition method.
\end{abstract}

PACS numbers: 42.70.Qs,42.25.Bs,42.25.Fx

\section{INTRODUCTION}

As recognized even in the first papers on the subject $[1,2]$, many of the most important applications of photonic crystals (PCs) make use of defect states which lie within a photonic band gap. Defects can be formed by a variety of microlithographic techniques $[3,4]$, and are important not only for controlling the propagation of light, but also for applications such as sensing and nonlinear switching [5]. Although many fully numerical studies have been undertaken [6], shallow defects - those in the vicinity of the band-edges where the perturbation is small - are difficult to model accurately even with powerful computers, since their fields extend very large distances from the defect. For these situations asymptotic methods are more appropriate and powerful, and have previously been used to study perturbations of dielectric interfaces in electromagnetism [7], strong localized perturbations in finite structures [8] and photonic crystals consisting of arrays of highly-conducting inclusions [9]. In these previous studies the structure was either finite in extent or the perturbation ranged over the entire photonic crystal. Defect states, however, result from a localized perturbation of an infinitely extended Bloch mode. How these localized states evolve from the band edge as a function of the strength of the perturbation is the issue we address here.

We present here a thorough discussion of the analytic properties of defects near the band-edge of a twodimensional PC. Our approach is an extension of the method first reported in Ref. [10], and is based on a Bloch function expansion of the Green's function in a PC [11]. We find that a localized perturbation away from the band-edge leads to interesting asymptotic behaviour, in that the highest-order change in frequency displays an exponential dependence on the magnitude of

*Electronic address: Kokou.Dossou@uts.edu.au the perturbation - specifically, the relative change in the electric energy caused by the perturbation - and also on the density of states of the unperturbed photonic crystal. This complements standard perturbation theory [7], in which the change in frequency depends linearly on the relative change of the electric energy caused by the perturbation. The asymptotic behaviour that we report is the result of the transition from an infinitely extended (Bloch) mode on the band-edge to a localized state in the gap. We also derive the leading order asymptotic expression for the spatial field profile of the defect state, and show that it takes the form of a combination of Bloch functions on the band extrema, multiplied by an exponential function.

Defects can be created in different ways, and so we study several cases in order to demonstrate the generality of our results. In addition to studying the two principal polarizations, we investigate defects arising from a change in the dielectric constant of a single cylinder, the variation of its radius, and from the application of a metallic coating. It is important to note that the asymptotic method we derive here is intended as an interpretive tool, useful for investigating the physics behind defect mode creation and evolution. Quantitative predictions must of course rely on more cumbersome numerical simulations that solve the electromagnetic problem in its entirety. Nevertheless, the results derived here should give accurate results close to the band edge. We check our analytic expressions by comparing them with numerical results based on the Fictitious Sources Superposition (FSS) Method [12-14], a technique which is well adapted to the accurate calculation of the properties of even highly extended defect modes. Though, in our examples, we use a PC constructed from uniform cylinders in an otherwise uniform background, the analytic results apply to all two-dimensional photonic crystals, irrespective of the geometry.

Our results show that the symmetries of the Bloch modes at band extrema play an important role in the creation and evolution of defect states, leading to three 
broad classes of defect dispersion relations. The first of these results in a nondegenerate dispersion relation. In the second, the dispersion relations are doubly degenerate, and derive from a two-fold degeneracy of the underlying Bloch function. The third, and most interesting, case occurs when the underlying Bloch function cannot be made real, such as occurs at the $\mathrm{K}$ point in the Brillouin zone of the hexagonal lattice. In this case, there are two defect modes with different dispersion relations which are both exponentially weakly bound and have the same cutoff.

We commence in Section II with a discussion of the Bloch mode representation of the photonic crystal Green's function, and follow this, in Section III, with the analysis of the behaviour of Bloch modes near the band edge and the consequences of this for the asymptotic behaviour of the Green's function. We then derive a convenient Bessel function representation which encapsulates the spatial decay of the defect Green's function. In Section IV, we apply the asymptotic form of the Green's function to derive a first-order expression for how the defect mode frequency depends on the magnitude of the perturbation. Results are given for the two principal polarizations. Associated with this analysis are a number of mathematical and physical subtleties which are discussed in the Appendix. Section IV also compares the results of the asymptotic theory with rigorous numerical calculations and we conclude with a discussion of the results presented.

\section{GREEN'S FUNCTION}

We consider a two-dimensional (2D) PC characterized by a periodic relative permittivity $\varepsilon(\boldsymbol{r})=n^{2}(\boldsymbol{r})$ (where $n(\boldsymbol{r})$ denotes the refractive index) and relative permeability $\mu(\boldsymbol{r})=1$. To construct a defect, we locally perturb the permittivity and define $\tilde{\varepsilon}(\boldsymbol{r})$ to be the permittivity distribution associated with the defect structure. All permittivities are taken to be real, making the PC lossless and the subsequent mathematical analysis selfadjoint. We further assume that the band-gaps in which we compute defect modes are complete (rather than partial) band-gaps. The defect mode, $\psi(\boldsymbol{r})$, satisfies the polarization dependent boundary value problem

$$
\tilde{\mathcal{L}} \psi+\frac{\omega^{2}}{c^{2}} \tilde{s}(\boldsymbol{r}) \psi(\boldsymbol{r})=0
$$

where

$$
\tilde{\mathcal{L}} \psi \equiv \nabla \cdot(\tilde{p}(\boldsymbol{r}) \nabla \psi(\boldsymbol{r})) .
$$

By writing the wave equation in this way, we explicitly encapsulate the continuity conditions for each polarization through the appropriate choice of the functions $\tilde{p}(\boldsymbol{r})$ and $\tilde{s}(\boldsymbol{r})$. For TM $\left(E_{\|}\right)$polarization, in which the electric field is parallel to the cylinder axes, $\psi(\boldsymbol{r})=E_{z}$ and we have $\tilde{p}(\boldsymbol{r})=1, \tilde{s}(\boldsymbol{r})=\tilde{\varepsilon}(\boldsymbol{r})$, while for $\mathrm{TE}\left(H_{\|}\right)$ polarization, in which the magnetic field is parallel to the cylinders and $\psi(\boldsymbol{r})=H_{z}$, we have $\tilde{p}(\boldsymbol{r})=1 / \tilde{\varepsilon}(\boldsymbol{r})$, $\tilde{s}(\boldsymbol{r})=1$. Since we assume that $\tilde{\varepsilon}$ is real, and since also the fields decay with increasing distance from the defect (a consequence of working in a full band-gap), it follows that both $\psi(\boldsymbol{r})$ and $\psi^{*}(\boldsymbol{r})$ are solutions of the boundary value problem (1), with this, in turn, implying that the defect mode $\psi$ may be taken to be a real valued function.

In order to analyze the defect states, we first formulate the Green's function $G\left(\boldsymbol{r}, \boldsymbol{r}^{\prime} ; \omega\right)$, which characterizes the response of the system at position $\boldsymbol{r}$ to a point source radiating with an angular frequency of $\omega$ at position $\boldsymbol{r}^{\prime}$. The Green's function satisfies

$$
\mathcal{L} G\left(\boldsymbol{r}, \boldsymbol{r}^{\prime} ; \omega\right)+\frac{\omega^{2}}{c^{2}} s\left(\boldsymbol{r}^{\prime}\right) G\left(\boldsymbol{r}, \boldsymbol{r}^{\prime} ; \omega\right)=\delta\left(\boldsymbol{r}-\boldsymbol{r}^{\prime}\right),
$$

and decays exponentially with increasing distance from the defect since the frequency $\omega$ lies inside a band gap. Here, we define the operator $\mathcal{L}$ by

$$
\mathcal{L} \varphi \equiv \nabla \cdot(p(\boldsymbol{r}) \nabla \varphi(\boldsymbol{r})),
$$

in which $p(\boldsymbol{r})$ and $s(\boldsymbol{r})$ are the analogues of $\tilde{p}(\boldsymbol{r})$ and $\tilde{s}(\boldsymbol{r})$ for the unperturbed, periodic structure. Since we are considering defect modes created within a complete band gap, the local density of states is zero over the WignerSeitz cell (WSC), and so the Green's function must be real.

Following the treatment of Ref. [11], we express $G\left(\boldsymbol{r}, \boldsymbol{r}^{\prime} ; \omega\right)$ as a superposition of quasiperiodic Green's functions $G\left(\boldsymbol{r}, \boldsymbol{r}^{\prime} ; \omega, \boldsymbol{k}_{0}\right)$ satisfying

$$
\begin{array}{r}
\mathcal{L} G\left(\boldsymbol{r}, \boldsymbol{r}^{\prime} ; \omega, \boldsymbol{k}_{0}\right)+\frac{\omega^{2}}{c^{2}} s\left(\boldsymbol{r}^{\prime}\right) G\left(\boldsymbol{r}, \boldsymbol{r}^{\prime} ; \omega, \boldsymbol{k}_{0}\right) \\
=\sum_{l} \delta\left(\boldsymbol{r}-\boldsymbol{r}^{\prime}-\boldsymbol{R}_{l}\right) e^{i \boldsymbol{k}_{0} \cdot \boldsymbol{R}_{l}}
\end{array}
$$

and associated with the (real) Bloch vector $\boldsymbol{k}_{0} \in \mathrm{BZ}$, where BZ denotes the (first) Brillouin zone of the reciprocal lattice. In Eq. (5), the sum extends over all direct lattice vectors $\boldsymbol{R}_{l}$ in the infinite PC. The computational examples that we consider in this paper are for regular two-dimensional lattices characterized by a pitch $d$.

It is then straightforward to construct $G\left(\boldsymbol{r}, \boldsymbol{r}^{\prime} ; \omega\right)$ by integrating $G\left(\boldsymbol{r}, \boldsymbol{r}^{\prime} ; \omega, \boldsymbol{k}_{0}\right)$ over the Brillouin zone, noting that by averaging over the BZ the source term on the right hand side of Eq. (5) reduces to only the primary source of Eq. (3) since

$$
\int_{\mathrm{BZ}} e^{i \boldsymbol{k}_{0} \cdot \boldsymbol{R}_{l}} d^{2} \boldsymbol{k}_{0}= \begin{cases}A_{\mathrm{BZ}}, & \text { if } \boldsymbol{R}_{l}=0 \\ 0, & \text { otherwise }\end{cases}
$$

where $A_{\mathrm{B} Z}$ is the area of the Brillouin Zone.

The important intermediate step of constructing the quasiperiodic Green's function $G\left(\boldsymbol{r}, \boldsymbol{r}^{\prime} ; \omega, \boldsymbol{k}_{0}\right)$ requires its expansion in a basis of the Bloch modes $\psi\left(\boldsymbol{k}_{0}, \boldsymbol{r}\right)$ of the bulk PC. These satisfy the generalized Helmholtz equation

$$
\mathcal{L} \psi\left(\boldsymbol{k}_{0}, \boldsymbol{r}\right)=-\frac{\omega^{2}}{c^{2}} s(\boldsymbol{r}) \psi\left(\boldsymbol{k}_{0}, \boldsymbol{r}\right)
$$


together with the Bloch condition

$$
\psi\left(\boldsymbol{k}_{0}, \boldsymbol{r}+\boldsymbol{R}_{l}\right)=\psi\left(\boldsymbol{k}_{0}, \boldsymbol{r}\right) e^{i \boldsymbol{k}_{0} \cdot \boldsymbol{R}_{l}} .
$$

For real $\varepsilon(\boldsymbol{r})$ (and hence real $p(\boldsymbol{r})$ and $s(\boldsymbol{r})$ ), the operator $\mathcal{L}$ is Hermitian with respect to the inner product

$$
\langle\phi, \psi\rangle=\frac{1}{A_{\mathrm{WSC}}} \int_{\mathrm{WSC}} s(\boldsymbol{r}) \psi\left(\boldsymbol{k}_{0}, \boldsymbol{r}\right) \phi^{*}\left(\boldsymbol{k}_{0}, \boldsymbol{r}\right) d^{2} \boldsymbol{r},
$$

in which the integration is evaluated over the WSC. The eigenfunctions $\left\{\psi_{m}\right\}$, corresponding to the countably infinite set of eigenfrequencies $\left\{\omega_{m}\right\}$, are then orthogonal with respect to this inner product and are normalized according to

$$
\left\langle\psi_{l}, \psi_{m}\right\rangle=M_{l} \delta_{l, m}, \text { where } M_{l}=\left\langle\psi_{l}, \psi_{l}\right\rangle \text {. }
$$

Then, $G\left(\boldsymbol{r}, \boldsymbol{r}^{\prime} ; \omega, \boldsymbol{k}_{0}\right)$ may be expanded in a series of Bloch modes $\left\{\psi_{m}\right\}$, as may also the source term on the right hand side of Eq. (5), using Poisson's summation formula:

$$
\begin{aligned}
& \sum_{l=-\infty}^{\infty} \delta\left(\boldsymbol{r}-\boldsymbol{r}^{\prime}-\boldsymbol{R}_{l}\right) e^{i \boldsymbol{k}_{0} \cdot \boldsymbol{R}_{l}} \\
= & \frac{1}{A_{\mathrm{WSC}}} \sum_{l} \frac{1}{M_{l}} s(\boldsymbol{r}) \psi_{l}\left(\boldsymbol{k}_{0}, \boldsymbol{r}\right) \psi_{l}^{*}\left(\boldsymbol{k}_{0}, \boldsymbol{r}^{\prime}\right) .
\end{aligned}
$$

Proceeding in this way, we derive the following expression for the quasiperiodic Green's function

$$
G\left(\boldsymbol{r}, \boldsymbol{r}^{\prime} ; \omega, \boldsymbol{k}_{0}\right)=\frac{c^{2}}{A_{\mathrm{WSC}}} \sum_{l} \frac{1}{M_{l}} \frac{\psi_{l}\left(\boldsymbol{k}_{0}, \boldsymbol{r}\right) \psi_{l}^{*}\left(\boldsymbol{k}_{0}, \boldsymbol{r}^{\prime}\right)}{\omega^{2}-\omega_{l}^{2}\left(\mathbf{k}_{0}\right)},
$$

and finally, by BZ averaging, we deduce

$$
G\left(\boldsymbol{r}, \boldsymbol{r}^{\prime} ; \omega\right)=\frac{c^{2}}{4 \pi^{2}} \sum_{l} \int_{\mathrm{BZ}} \frac{1}{M_{l}} \frac{\psi_{l}\left(\boldsymbol{k}_{0}, \boldsymbol{r}\right) \psi_{l}^{*}\left(\boldsymbol{k}_{0}, \boldsymbol{r}^{\prime}\right)}{\omega^{2}-\omega_{l}^{2}\left(\mathbf{k}_{0}\right)} d^{2} \boldsymbol{k}_{0}
$$

noting that $A_{\mathrm{WSC}} A_{\mathrm{BZ}}=4 \pi^{2}$ in $2 \mathrm{D}$ lattices.

\section{ASYMPTOTIC BEHAVIOUR OF THE GREEN'S FUNCTION NEAR THE GAP EDGE}

Having established the form of the Green's function (13), we now consider its behaviour for a frequency $\omega$ in the vicinity of the edge of a particular band $l=L$ which occurs at one, or more, positions in the BZ, $\boldsymbol{k}_{0}=\boldsymbol{k}_{L, j}$, for $j=1,2, \ldots, \nu_{L}$. We begin with the simplest case in which the band extremum occurs at a single point contained completely within the BZ, and generalize this later to handle the most common case in which the band has multiple extrema that typically occur at the symmetry points on the BZ boundary. (a)
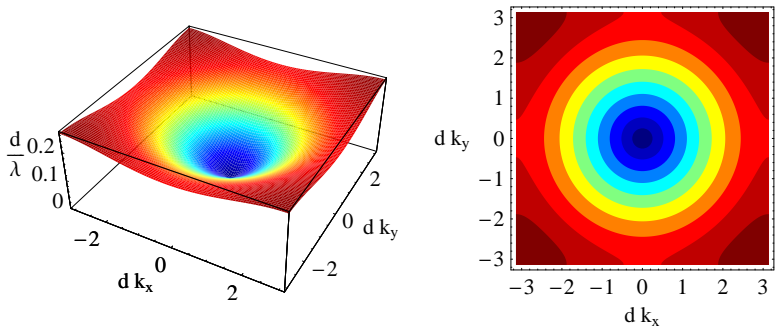

(b)
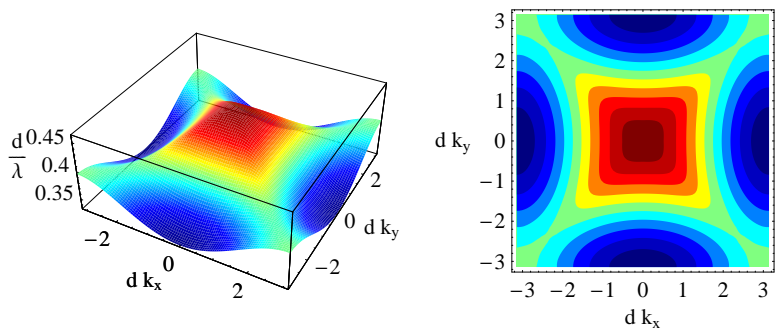

FIG. 1: (Color online) Band surfaces for a square rod-type $\mathrm{PC}$ with $n_{b}=1, n_{c}=3$ and $a / d=0.3$ operated in TM polarization. (a) The first band is rotationally symmetric near the two band edges, $\Gamma$ (the BZ centre) and $M$ (BZ corners). (b) The second band has an elliptic profile near the lower edge ( $X$-the middle of the BZ sides). The vertical axis shows the normalized frequency $d / \lambda=\omega d /(2 \pi c)$.

In our treatment, we assume that the band extremum at $\left(\boldsymbol{k}_{L}, \omega\right)$ exhibits a generic paraboloidal shape

$$
\begin{aligned}
\omega_{L}\left(\boldsymbol{k}_{0}\right)= & \omega_{L}+\frac{1}{2 C_{L, x}}\left(k_{0, x}-k_{L, x}\right)^{2} \\
& +\frac{1}{2 C_{L, y}}\left(k_{0, y}-k_{L, y}\right)^{2}+O\left(\left\|\boldsymbol{k}_{0}-\boldsymbol{k}_{L}\right\|^{4}\right) \\
=\omega_{L} & +\frac{1}{2 C_{L}}\left\|\boldsymbol{\sigma}\left(\boldsymbol{k}_{0}-\boldsymbol{k}_{L}\right)\right\|^{2}+O\left(\left\|\boldsymbol{k}_{0}-\boldsymbol{k}_{L}\right\|^{4}\right) .(1
\end{aligned}
$$

The constants $C_{L, x}$ and $C_{L, y}$ characterize the band curvatures in the $k_{x}$ and $k_{y}$ directions of the BZ. In (15), we simplify the notation through the introduction of $C_{L}$, denoting the geometric mean $\left(C_{L}=\operatorname{sign} C_{L, x} \times\right.$ $\left.\sqrt{C_{L, x} C_{L, y}}\right)$ of the two curvatures, and a scaling matrix $\boldsymbol{\sigma}=\operatorname{diag}\left\{\left(C_{L, y} / C_{L, x}\right)^{1 / 4},\left(C_{L, x} / C_{L, y}\right)^{1 / 4}\right\}$. For a circular parabolic band surface, $C_{L}=C_{L, x}=C_{L, y}$, and $\boldsymbol{\sigma}=\boldsymbol{I}$, the identity transformation. This is exemplified in Figs 1 (a) and (b) which show the first two band surfaces for a square lattice PC. In Fig. 1 (a), we see that $C_{L, x}=C_{L, y}$ for both edges of the first band which occur at $\Gamma$ and $X$ in the BZ. However, in Fig. 1 (b) we see that $C_{L, x} \neq C_{L, y}$ near the $X$-points on the lower edge of the second band since the band curvatures in the $k_{x}$ and $k_{y}$ directions differ.

We now consider the asymptotic analysis of the Green's function (13) when the frequency $\omega$ approaches the band edge from within the gap - corresponding to frequencies in the band gap which are either just below or just above 
the edge of band $l=L$. Term $l=L$ of the series (13) for $G$ now dominates the expansion and so we write

$$
G\left(\boldsymbol{r}, \boldsymbol{r}^{\prime} ; \omega\right)=G_{L}\left(\boldsymbol{r}, \boldsymbol{r}^{\prime} ; \omega\right)+\sum_{l \neq L} G_{l}\left(\boldsymbol{r}, \boldsymbol{r}^{\prime} ; \omega\right)
$$

separating out the contributions from the various terms $l$. Since $\omega$ is not in the vicinity of any band $l \neq L$, then the sum over these terms in Eq. (16) is a smooth function of $\omega$ at the gap edge, and of magnitude $O\left(\left|\omega-\omega_{L}\right|^{0}\right)$.

The analysis for the term $G_{L}$ requires some delicacy since $\omega \approx \omega_{L}$. Here, we substitute the band surface approximation (15) into term $L$ of Eq. (13). Neglecting terms of $O\left(\left\|\boldsymbol{k}_{0}-\boldsymbol{k}_{L}\right\|^{4}\right)$, we have

$$
\begin{aligned}
& G_{L}\left(\boldsymbol{r}, \boldsymbol{r}^{\prime} ; \omega\right)= \\
& \frac{c^{2}}{8 \pi^{2} \omega} \int_{\mathrm{BZ}} \frac{1}{M_{L}} \frac{\psi_{L}\left(\boldsymbol{k}_{0}, \boldsymbol{r}\right) \psi_{L}^{*}\left(\boldsymbol{k}_{0}, \boldsymbol{r}^{\prime}\right)}{\omega-\omega_{L}-\frac{1}{2 C_{L}}\left\|\boldsymbol{\sigma}\left(\boldsymbol{k}_{0}-\boldsymbol{k}_{L}\right)\right\|^{2}} d^{2} \boldsymbol{k}_{0} \\
& +O\left(\left\|\boldsymbol{k}_{0}-\boldsymbol{k}_{L}\right\|^{0}\right) .
\end{aligned}
$$

To simplify this further, for computational purposes, we express the Bloch functions as the product $\psi_{L}\left(\boldsymbol{k}_{0}, \boldsymbol{r}\right)=$ $u_{L}\left(\boldsymbol{k}_{0}, \boldsymbol{r}\right) \exp \left(i \boldsymbol{k}_{0} \cdot \boldsymbol{r}\right)$, where $u_{L}$ is a periodic function. The singularity in the denominator of the integrand of Eq. (17) ensures that, for $\omega$ in the vicinity of the band surface, only those $\boldsymbol{k}_{0}$ in the vicinity of $\boldsymbol{k}_{L}$ contribute significantly to the integral. Accordingly, we write $\psi_{L}\left(\boldsymbol{k}_{0}, \boldsymbol{r}\right) \approx \psi_{L}\left(\boldsymbol{k}_{L}, \boldsymbol{r}\right) \exp \left(i\left(\boldsymbol{k}_{0}-\boldsymbol{k}_{L}\right) \cdot \boldsymbol{r}\right)$, thus leading to the following form

$$
\begin{aligned}
& G_{L}\left(\boldsymbol{r}, \boldsymbol{r}^{\prime} ; \omega\right) \approx \frac{c^{2} \psi_{L}\left(\boldsymbol{k}_{L}, \boldsymbol{r}\right) \psi_{L}^{*}\left(\boldsymbol{k}_{L}, \boldsymbol{r}^{\prime}\right)}{8 \pi^{2} \omega M_{L}} \\
& \quad \times \int_{\mathrm{BZ}} \frac{e^{i\left(\boldsymbol{k}_{0}-\boldsymbol{k}_{L}\right) \cdot\left(\boldsymbol{r}-\boldsymbol{r}^{\prime}\right)}}{\delta \omega-\frac{1}{2 C_{L}}\left\|\boldsymbol{\sigma}\left(\boldsymbol{k}_{0}-\boldsymbol{k}_{L}\right)\right\|^{2}} d^{2} \boldsymbol{k}_{0}+O\left((\delta \omega)^{0}\right),
\end{aligned}
$$

with

$$
\delta \omega=\omega-\omega_{L}
$$

The integral in Eq. (18) may be further simplified by noting that when $\omega$ is near the band edge, the singularity dominates the integral, allowing the integral over the Brillouin zone to be replaced by an integral over the entire k-plane. In this, we follow the classical treatment of electrons in perturbed periodic potentials developed by Luttinger and Kohn $[15,16]$. Thus,

$$
\begin{array}{r}
\int_{\mathrm{BZ}} \frac{e^{i\left(\boldsymbol{k}_{0}-\boldsymbol{k}_{L}\right) \cdot\left(\boldsymbol{r}-\boldsymbol{r}^{\prime}\right)}}{\delta \omega-\frac{1}{2 C_{L}}\left\|\boldsymbol{\sigma}\left(\boldsymbol{k}_{0}-\boldsymbol{k}_{L}\right)\right\|^{2}} d^{2} \boldsymbol{k}_{0} \approx \\
\int_{\mathbb{R}^{2}} \frac{e^{i \boldsymbol{\sigma}\left(\boldsymbol{k}_{0}-\boldsymbol{k}_{L}\right) \cdot \boldsymbol{\sigma}^{-1}\left(\boldsymbol{r}-\boldsymbol{r}^{\prime}\right)}}{\delta \omega-\frac{1}{2 C_{L}}\left\|\boldsymbol{\sigma}\left(\boldsymbol{k}_{0}-\boldsymbol{k}_{L}\right)\right\|^{2}} d^{2} \boldsymbol{\sigma} \boldsymbol{k}_{0}+O\left((\delta \omega)^{0}\right) .
\end{array}
$$

In deriving this form, we have changed the integration variable from $\boldsymbol{k}_{0}$ to $\boldsymbol{\sigma} \boldsymbol{k}_{0}$, noting that the Jacobian of the transformation is unity since $\operatorname{det} \boldsymbol{\sigma}=1$. Then, from the integral representation for the zeroth order MacDonald function $K_{0}$, i.e., a modified Bessel function of the second kind

$$
\int_{\mathbb{R}^{2}} \frac{e^{i \boldsymbol{k}_{0} \cdot\left(\boldsymbol{r}-\boldsymbol{r}^{\prime}\right)}}{k^{2}+\left|\boldsymbol{k}_{0}\right|^{2}} d^{2} \boldsymbol{k}_{0}=2 \pi K_{0}\left(k\left|r-r^{\prime}\right|\right),
$$

we arrive at

$$
\begin{gathered}
\int_{\mathrm{BZ}} \frac{e^{i\left(\boldsymbol{k}_{0}-\boldsymbol{k}_{L}\right) \cdot\left(\boldsymbol{r}-\boldsymbol{r}^{\prime}\right)}}{\delta \omega-\frac{1}{2 C_{L}}\left\|\boldsymbol{\sigma}\left(\boldsymbol{k}_{0}-\boldsymbol{k}_{L}\right)\right\|^{2}} d^{2} \boldsymbol{k}_{0}= \\
=-4 \pi C_{L} K_{0}\left(b\left|\boldsymbol{\sigma}^{-1}\left(\boldsymbol{r}-\boldsymbol{r}^{\prime}\right)\right|\right)+O\left((\delta \omega)^{0}\right) .
\end{gathered}
$$

Here,

$$
b=\sqrt{-2\left|C_{L}\right| s_{L} \delta \omega},
$$

in which $s_{L}=\operatorname{sign} C_{L}$, with the significance of this latter quantity becoming apparent in the discussion leading to the development of Eq. (51) in Section IV B. Here, we simply observe that the quantity $s_{L} \delta \omega<0$, which is consistent with the defect mode occurring in the band-gap either above a band surface maximum $\left(s_{L}=-1\right)$ or below a minimum $\left(s_{L}=+1\right)$. The integral representation for the MacDonald function (21) can be derived directly from a similar expression given on p. 817 in Ref. [17] for the Hankel function of the first kind, $H_{0}^{(1)}$, and the relation $K_{0}(z)=\frac{1}{2} \pi i H_{0}^{(1)}(i z)$.

Before studying the asymptotics of the evolution of the defect mode from the band edge, we generalize our treatment to accommodate the common situation in which a band has multiple extrema at $\boldsymbol{k}_{0}=\boldsymbol{k}_{L, j}$ for $j=1,2, \ldots, \nu_{L}$. These extrema are typically high symmetry points in the BZ; this is shown in Fig. 1, which presents band surfaces for a square lattice, having multiple extrema at the BZ edge at the points $X$ and $M$.

The treatment proceeds as before, except now we regard the band surface as being locally paraboloidal about each of the extrema $\boldsymbol{k}_{0}=\boldsymbol{k}_{L, j}$. Thus,

$$
G_{L}\left(\boldsymbol{r}, \boldsymbol{r}^{\prime} ; \omega\right)=\sum_{j=1}^{\nu_{L}} G_{L, j}\left(\boldsymbol{r}, \boldsymbol{r}^{\prime} ; \omega\right),
$$

where

$$
\begin{aligned}
& G_{L, j}\left(\boldsymbol{r}, \boldsymbol{r}^{\prime} ; \omega\right)=\frac{c^{2} \psi_{L}\left(\boldsymbol{k}_{L, j}, \boldsymbol{r}\right) \psi_{L}^{*}\left(\boldsymbol{k}_{L, j}, \boldsymbol{r}^{\prime}\right)}{8 \pi^{2} \omega M_{L}} \\
& \left.\times \int_{\mathrm{BZ}} \frac{e^{i\left(\boldsymbol{k}_{0}-\boldsymbol{k}_{L, j}\right) \cdot\left(\boldsymbol{r}-\boldsymbol{r}^{\prime}\right)}}{\delta \omega-\frac{1}{2 C_{L}}\left\|\boldsymbol{\sigma}\left(\boldsymbol{k}_{0}-\boldsymbol{k}_{L, j}\right)\right\|^{2}} d^{2} \boldsymbol{k}_{0}+O(\delta \omega)^{0}\right),
\end{aligned}
$$

which follows from Eq. (18), with $M_{L, j}=M_{L}$ and $C_{L, j}=C_{L}$, for all $j$, due to symmetry. With several band extrema located on the boundary of the Brillouin zone, the integral over the Brillouin zone must be modified in such a way as to account correctly for these multiple extrema, each of which contribute to a portion of the integral. Eq. (22) is thus modified to

$$
\begin{array}{r}
\int_{\mathrm{BZ}} \frac{e^{i\left(\boldsymbol{k}_{0}-\boldsymbol{k}_{L}\right) \cdot\left(\boldsymbol{r}-\boldsymbol{r}^{\prime}\right)}}{\delta \omega-\frac{1}{2 C_{L}}\left\|\boldsymbol{\sigma}\left(\boldsymbol{k}_{0}-\boldsymbol{k}_{L}\right)\right\|^{2}} d^{2} \boldsymbol{k}_{0}=-4 \pi \theta_{L} C_{L} \\
\times K_{0}\left(b\left|\boldsymbol{\sigma}^{-1}\left(\boldsymbol{r}-\boldsymbol{r}^{\prime}\right)\right|\right)+O\left((\delta \omega)^{0}\right),
\end{array}
$$


where $2 \pi \theta_{L}$ is the interior angle of the BZ at each point $\boldsymbol{k}_{0}=\boldsymbol{k}_{L, j}$.

Finally, we obtain the leading order estimate for the Green's function from Eqs (24)-(26)

$$
\begin{aligned}
G\left(\boldsymbol{r}, \boldsymbol{r}^{\prime}\right. & ; \omega)=-\frac{c^{2} C_{L} \theta_{L}}{2 \pi \omega_{L} M_{L}} K_{0}\left(b\left|\boldsymbol{\sigma}\left(\boldsymbol{r}-\boldsymbol{r}^{\prime}\right)\right|\right) \\
& \times \sum_{j=1}^{\nu_{L}} \psi_{L}\left(\boldsymbol{k}_{L, j}, \boldsymbol{r}\right) \psi_{L}^{*}\left(\boldsymbol{k}_{L, j}, \boldsymbol{r}^{\prime}\right)+O\left((\delta \omega)^{0}\right) .
\end{aligned}
$$

Since the Green's function $G\left(\boldsymbol{r}, \boldsymbol{r}^{\prime} ; \omega\right)$ must be real, as argued above, we require that its approximation (27) involving Bloch mode expansions be real valued. The imaginary part of the sum in Eq. (27) can be made to vanish either if the functions $\left\{\psi_{L}\left(\boldsymbol{k}_{L, j}, \boldsymbol{r}\right)\right\}$ can be scaled to be real functions or, if this is not possible, by ensuring that pairs of "opposite" Bloch functions, $\psi_{L}\left(\boldsymbol{k}_{L, j}, \boldsymbol{r}\right)$ and $\psi_{L}\left(-\boldsymbol{k}_{L, j}, \boldsymbol{r}\right)$, are scaled so that $\psi_{L}\left(-\boldsymbol{k}_{L, j}, \boldsymbol{r}\right)=$ $\psi_{L}^{*}\left(\boldsymbol{k}_{L, j}, \boldsymbol{r}\right)$. From the representation of a Bloch mode $\psi_{L}(\boldsymbol{k}, \boldsymbol{r})$ as the product of a periodic function and the Bloch factor $e^{i \boldsymbol{k} \cdot \boldsymbol{r}}$, it follows that $\psi_{L}^{*}(\boldsymbol{k}, \boldsymbol{r})$ must be the Bloch mode that is associated with the Bloch vector $\boldsymbol{- k}$. Thus, it is easy to see that contributions to the series in Eq. (27) due to "opposite" pairs lead to a sum of two conjugate pairs, resulting in a real quantity.

In the following analysis, we require two asymptotic forms for the Green's function. The first of these, in which $b$ is small and $\left|\boldsymbol{r}-\boldsymbol{r}^{\prime}\right|=O(d)$, (i.e., of the order of the lattice constant) arises in the derivation of the dispersion relation for the defect mode and relies on the small argument asymptotic form: $K_{0}(z)=-\ln z+\ln 2-$ $\gamma+O\left(z^{2}\right)$, for $z \ll 1$. The Green's function is then approximated by

$$
\begin{aligned}
G\left(\boldsymbol{r}, \boldsymbol{r}^{\prime} ; \omega\right) \approx & \frac{c^{2} C_{L} \theta_{L}}{4 \pi \omega_{L} M_{L}}\left(\ln |\delta \omega|+C_{0}\right) \times \\
& \times \sum_{j=1}^{\nu_{L}} \psi_{L}\left(\boldsymbol{k}_{L, j}, \boldsymbol{r}\right) \psi_{L}^{*}\left(\boldsymbol{k}_{L, j}, \boldsymbol{r}^{\prime}\right),
\end{aligned}
$$

in which $C_{0}$ contains higher order terms in $|\delta \omega|$. The second form occurs in the construction of the extended form of the defect mode and applies when $b$ is small, yet $z=b\left|\boldsymbol{r}-\boldsymbol{r}^{\prime}\right|$ is large. In this large argument approximation, we have $K_{0}(z) \approx \sqrt{\pi} \exp (-z) / \sqrt{2 z}$, with this leading to the following asymptotic form of the Green's function:

$$
\begin{aligned}
G\left(\boldsymbol{r}, \boldsymbol{r}^{\prime} ; \omega\right) \approx & -\frac{c^{2} C_{L} \theta_{L} \sqrt{2 \pi}}{4 \pi \omega_{L} M_{L}} \frac{e^{-b\left|\boldsymbol{r}-\boldsymbol{r}^{\prime}\right|}}{\sqrt{b\left|\boldsymbol{r}-\boldsymbol{r}^{\prime}\right|}} \\
& \times \sum_{j=1}^{\nu_{L}} \psi_{L}\left(\boldsymbol{k}_{L}, \boldsymbol{r}\right) \psi_{L}^{*}\left(\boldsymbol{k}_{L, j}, \boldsymbol{r}^{\prime}\right) .
\end{aligned}
$$

This representation of the Green's function ensures that the defect mode decays exponentially with a rate $b=$ $\sqrt{-2\left|C_{L}\right| s_{L} \delta \omega}$. Note that this rate corresponds to the results found by analytic continuation of the band into the gap [18].

\section{FORMULATION OF DEFECT MODES USING GREEN'S THEOREM}

Having developed expressions for the Green's function we now turn to their use in defect mode calculations.

\section{A. General results}

We exploit the Green's functions (27) and (28) to find the mode of a single defect in an infinite PC for a frequency $\omega$ within a band gap in the vicinity of band $L$. At the point at which the defect mode originates from the band surface extrema, it is determined by a linear combination of the associated Bloch functions of the infinite $\mathrm{PC}$ and so is arbitrarily extended. As the mode evolves into the gap, the mode decays exponentially with increasing distance from the defect. However, it remains well approximated by a Bloch mode, or a linear combination thereof, in the vicinity of the defect, provided that $b(23)$ is sufficiently small. Since, however, the actual defect mode must be real valued, it is necessary to impose conditions that ensure that the required linear combination of Bloch modes (which themselves are not necessarily real) is real.

The defect mode, $\psi(\boldsymbol{r})$, satisfies the generalized Helmholtz equation (1) and by applying Green's Theorem, we arrive at the exact and general expression for the spatial distribution of the defect mode

$$
\begin{aligned}
\psi(\boldsymbol{r})= & -\int_{\mathbb{R}^{2}}\left(p\left(\boldsymbol{r}^{\prime}\right)-\tilde{p}\left(\boldsymbol{r}^{\prime}\right)\right) \nabla^{\prime} G\left(\boldsymbol{r}, \boldsymbol{r}^{\prime} ; \omega\right) \cdot \nabla^{\prime} \psi\left(\boldsymbol{r}^{\prime}\right) d^{2} \boldsymbol{r}^{\prime} \\
& +\frac{\omega^{2}}{c^{2}} \int_{\mathbb{R}^{2}}\left(s\left(\boldsymbol{r}^{\prime}\right)-\tilde{s}\left(\boldsymbol{r}^{\prime}\right)\right) G\left(\boldsymbol{r}, \boldsymbol{r}^{\prime} ; \omega\right) \psi\left(\boldsymbol{r}^{\prime}\right) d^{2} \mathbf{r}^{\prime} .
\end{aligned}
$$

For TM polarization, $p(\boldsymbol{r})=\tilde{p}(\boldsymbol{r})=1$, and so only the second integral of Eq. (30) contributes to $\psi(\boldsymbol{r})$. Correspondingly, since $s(\boldsymbol{r})=\tilde{s}(\boldsymbol{r})=1$ for TE polarization, only the first integral of Eq. (30) contributes. Further, since the defect is of finite extent, $\delta p(\boldsymbol{r}) \equiv \tilde{p}(\boldsymbol{r})-p(\boldsymbol{r})$ and $\delta s(\boldsymbol{r}) \equiv \tilde{s}(\boldsymbol{r})-s(\boldsymbol{r})$, which respectively denote the change in the permittivity and inverse permittivity of the $\mathrm{PC}$, are non-zero only over a bounded region.

\section{B. Formulation of the modal dispersion equation}

We now formulate the dispersion equation of the defect mode, first considering TM polarization. From Eq. (30),

$$
\psi(\boldsymbol{r})=-\frac{\omega^{2}}{c^{2}} \int_{\mathbb{R}^{2}} \delta \varepsilon\left(\boldsymbol{r}^{\prime}\right) G\left(\boldsymbol{r}, \boldsymbol{r}^{\prime} ; \omega\right) \psi\left(\boldsymbol{r}^{\prime}\right) d^{2} \boldsymbol{r}^{\prime},
$$

where $\delta \varepsilon\left(\boldsymbol{r}^{\prime}\right)=\tilde{\varepsilon}\left(\boldsymbol{r}^{\prime}\right)-\varepsilon\left(\boldsymbol{r}^{\prime}\right)$. Then, into Eq. (31), we substitute the Green's function (28), retaining only the dominant frequency dependence which is of order $\ln |\delta \omega|$. We digress briefly to note that the terms that are omitted in this approximation are of order $O\left((\delta \omega)^{0}\right)$ and so contribute to the constant term (labelled $\mathcal{A}$ ) below. While 
these particular terms, associated with the contribution to $G$ from band $L$, can be included in a straightforward manner, there are also contributions to $G$ from other bands $l \neq L$ which contain terms of order $O\left((\delta \omega)^{0}\right)$. Their estimation is not straightforward and so we omit all but the dominant frequency dependent term, proceeding from here in the spirit of first order perturbation theory.

Thus, to leading order in $\delta \omega$, we have

$$
\begin{aligned}
\psi(\boldsymbol{r})= & -\frac{\omega_{L} C_{L} \theta_{L}}{4 \pi M_{L}} \ln |\delta \omega| \sum_{j=1}^{\nu_{L}}\left(\psi_{L}\left(\boldsymbol{k}_{L, j}, \boldsymbol{r}\right)\right. \\
& \left.\times \int_{\mathcal{D}_{0}} \delta \varepsilon\left(\boldsymbol{r}^{\prime}\right) \psi_{L}^{*}\left(\boldsymbol{k}_{L, j}, \boldsymbol{r}^{\prime}\right) \psi\left(\boldsymbol{r}^{\prime}\right) d^{2} \mathbf{r}^{\prime}\right),
\end{aligned}
$$

in which $\mathcal{D}_{0}$ denotes the defect region. Here, to first order, for weak perturbations, the defect mode is well approximated by either the relevant Bloch mode of the bulk PC, or some linear combination of Bloch modes associated with the band extrema.

While the analysis is quite straightforward when the band extremum is contained completely within the BZ, the treatment of the more common case, in which the band surface has multiple extreme points (at the edge of the BZ), is more complex and requires some understanding of the symmetry of the modes. The lattices with which we are concerned (i.e., square and hexagonal) are invariant under a rotation transformation $\mathcal{T}$ by an angle $2 \pi / \nu$ (e.g., $\pi / 3$ for a hexagonal lattice with $\nu=6)$. That is, $\varepsilon(\mathcal{T} \boldsymbol{r})=\varepsilon(\boldsymbol{r})$. Applying this transformation to the entire field problem, i.e., rotating both the lattice and the Bloch vector, the mode is invariant in that $\psi_{L}\left(\boldsymbol{k}_{0}, \boldsymbol{r}\right)=\psi_{L}\left(\boldsymbol{\mathcal { T }} \boldsymbol{k}_{0}, \boldsymbol{T} \boldsymbol{r}\right)$. This may be written in the alternative form $\psi_{L}\left(\boldsymbol{\mathcal { T }} \boldsymbol{k}_{0}, \boldsymbol{r}\right)=\psi_{L}\left(\boldsymbol{k}_{0}, \mathcal{T}^{T} \boldsymbol{r}\right)$, in which $\mathcal{T}^{-1}=\mathcal{T}^{T}$ since $\mathcal{T}$ is an orthogonal transformation. With these relations, we can replace all references in Eq. (32) to $\boldsymbol{k}_{L, j}=\boldsymbol{\mathcal { T }}_{j} \boldsymbol{k}_{L, 1}$ by $\boldsymbol{k}_{L, 1}$ where $\boldsymbol{\mathcal { T }}_{j}=(\boldsymbol{\mathcal { T }})^{j}$, denoting a rotation by $j$-times the canonical angle $2 \pi / \nu$.

Then, taking into account the fact that the integrands of the overlap integrals in Eq. (32) for TM polarization have the form $\left(\delta \varepsilon \boldsymbol{E}_{L}^{*} \cdot \boldsymbol{E}\right)$ where $\boldsymbol{E}_{L}=\psi_{L} \hat{z}$ and $\boldsymbol{E}=\psi \hat{z}$ are electric fields, Eq. (32) can be recast as

$$
\begin{array}{r}
\psi(\boldsymbol{r})=-\frac{\omega_{L} C_{L} A_{\mathrm{WSC}} \theta_{L}}{4 \pi \mathcal{E}_{L}} \ln |\delta \omega| \sum_{j=1}^{\nu_{L}}\left(\psi_{L}\left(\boldsymbol{k}_{L, 1}, \mathcal{T}_{j-1}^{T} \boldsymbol{r}\right)\right. \\
\left.\times \int_{\mathcal{D}_{0}} \delta \varepsilon\left(\boldsymbol{r}^{\prime}\right) \boldsymbol{E}_{L}^{*}\left(\boldsymbol{k}_{L, 1}, \mathcal{T}_{j-1}^{T} \boldsymbol{r}^{\prime}\right) \cdot \boldsymbol{E}\left(\boldsymbol{r}^{\prime}\right) d^{2} \boldsymbol{r}^{\prime}\right)(33)
\end{array}
$$

where $\mathcal{E}_{L}$ is the electric energy of the Bloch mode over the Wigner-Seitz cell:

$$
\mathcal{E}_{L}=\int_{\mathrm{WSC}} \varepsilon(\boldsymbol{r})\left\|\boldsymbol{E}_{L}\left(\boldsymbol{k}_{L, 1}, \boldsymbol{r}^{\prime}\right)\right\|^{2} d^{2} \boldsymbol{r}^{\prime}=A_{\mathrm{WSC}} M_{L}
$$

For TE polarization the derivation is similar to that for
TM polarization. The equation for TE polarization is

$$
\begin{gathered}
\psi(\boldsymbol{r})=\frac{c^{2} C_{L} \theta_{L}}{4 \pi \omega_{L} M_{L}} \ln |\delta \omega| \sum_{j=1}^{\nu_{L}}\left(\psi_{L}\left(\boldsymbol{k}_{L, 1}, \mathcal{T}_{j-1}^{T} \boldsymbol{r}\right)\right. \\
\left.\times \int_{\mathcal{D}_{0}} \delta \varepsilon^{-1}\left(\boldsymbol{r}^{\prime}\right) \nabla^{\prime} \psi_{L}^{*}\left(\boldsymbol{k}_{L, 1}, \boldsymbol{\mathcal { T }}_{j-1}^{T} \boldsymbol{r}^{\prime}\right) \cdot \nabla^{\prime} \psi\left(\boldsymbol{r}^{\prime}\right) d^{2} \boldsymbol{r}^{\prime}\right),
\end{gathered}
$$

where $\delta \varepsilon^{-1}(\boldsymbol{r}) \equiv \tilde{\varepsilon}^{-1}(\boldsymbol{r})-\varepsilon^{-1}(\boldsymbol{r})$. We can bring Eq. (35) for TE polarization into the same framework as Eq. (33) by noting that for TE polarization $\nabla \psi(\boldsymbol{r}) / \varepsilon(\boldsymbol{r})=-i \omega(\hat{z} \times \boldsymbol{E}(\boldsymbol{r}))$, where $\boldsymbol{E}$ is the transverse component of the electric field (perpendicular to the magnetic field $\boldsymbol{H}=\psi \hat{z}$ ). The integrands in Eq. (35) become

$-\delta \varepsilon\left(\boldsymbol{r}^{\prime}\right) \frac{\nabla^{\prime} \psi_{L}^{*}\left(\boldsymbol{k}_{L, 1}, \mathcal{T}_{j-1}^{T} \boldsymbol{r}^{\prime}\right)}{\varepsilon\left(\boldsymbol{r}^{\prime}\right)} \cdot \frac{\nabla^{\prime} \psi\left(\boldsymbol{r}^{\prime}\right)}{\tilde{\varepsilon}\left(\boldsymbol{r}^{\prime}\right)}=-\omega^{2} \delta \varepsilon \boldsymbol{E}_{L}^{*} \cdot \boldsymbol{F}$

In addition, the normalizing factor $M_{L}$ (10) is recast as

$$
M_{L}=\frac{1}{A_{\mathrm{WSC}}} \int_{\mathrm{WSC}}\left|\psi_{L}\left(\boldsymbol{k}_{L, 1}, \boldsymbol{r}^{\prime}\right)\right|^{2} d^{2} \boldsymbol{r}^{\prime}=\frac{c^{2}}{A_{\mathrm{WSC}}} \mathcal{E}_{L},
$$

which follows directly from the differential equation (7) and an application of Green's theorem.

Eqs (36) and (37) then lead immediately to precisely the same form as that derived for TM polarization (33). The unification of the formulation at this point simplifies the subsequent description of the analysis and the derivation of the defect mode dispersion calculations for both polarizations.

Eq. (33) is the last of the general results since, from here on, the analysis requires knowledge of the actual symmetry of the Bloch modes for each specific case. Our treatment here is motivated by the examples of the following section in which we study the evolution of modes from the upper and lower edges of band gaps for both square and hexagonal lattices. In its most general form, the analysis that leads to the derivation of the defect mode dispersion equation is quite complex and therefore many of the details are given in the Appendix. We now outline the key steps in the derivation that are required to deduce from Eq. (33) the results in Eq. (48) below.

The first step in the derivation is to approximate the defect mode as a linear combination of the Bloch modes $\mathcal{B}=\left\{\psi_{L}\left(\boldsymbol{k}_{L, 1}, \mathcal{T}_{j-1}^{T} \boldsymbol{r}\right)\right\}$, for $j=1, \ldots, \nu_{L}$. This set of modes, however, is not necessarily linearly independent. We thus draw from $\mathcal{B}$ a linearly independent set $\mathcal{L}$ of Bloch modes and, using expansion coefficients $t_{l}$, write the defect mode as the linear combination

$$
\psi(\boldsymbol{r}) \approx \sum_{l \in \mathcal{L}} t_{l} \psi_{L}\left(\boldsymbol{k}_{L, 1}, \boldsymbol{\mathcal { T }}_{l-1}^{T} \boldsymbol{r}\right)
$$

The electric field $\boldsymbol{E}(\boldsymbol{r})$ which appears in the overlap integrals of Eq. (33), is approximated using the same expansion coefficients $t_{l}$ as in Eq. (38)

$$
\boldsymbol{E}(\boldsymbol{r}) \approx \sum_{l \in \mathcal{L}} t_{l} \boldsymbol{E}_{L}\left(\boldsymbol{k}_{L, 1}, \boldsymbol{\mathcal { T }}_{l-1}^{T} \boldsymbol{r}\right)
$$


Note that for TM polarization Eqs (38) and (39) are identical while for TE polarization, Eq. (39) is equivalent to

$$
\frac{\nabla \psi(\boldsymbol{r})}{\tilde{\varepsilon}(\boldsymbol{r})} \approx \sum_{l \in \mathcal{L}} t_{l} \frac{\nabla \psi_{L}\left(\boldsymbol{k}_{L, 1}, \boldsymbol{\mathcal { T }}_{l-1}^{T} \boldsymbol{r}\right)}{\varepsilon(\boldsymbol{r})} .
$$

Then, substituting Eqs (38) and (39) into Eq. (33), we deduce that for either polarization

$$
\begin{aligned}
& \sum_{l \in \mathcal{L}} t_{l} \psi_{L}\left(\boldsymbol{k}_{L, 1}, \mathcal{T}_{l-1}^{T} \boldsymbol{r}\right)=-\frac{\omega_{L} C_{L} A_{\mathrm{WSC}} \theta_{L}}{4 \pi \mathcal{E}_{L}} \\
& \times \ln |\delta \omega| \sum_{l \in \mathcal{L}} t_{l} \sum_{j=1}^{\nu_{L}} B_{l, j} \psi_{L}\left(\boldsymbol{k}_{L, 1}, \mathcal{T}_{j-1}^{T} \boldsymbol{r}\right),
\end{aligned}
$$

in which

$$
B_{l, j}=\int_{\mathcal{D}_{0}} \delta \varepsilon\left(\boldsymbol{r}^{\prime}\right) \boldsymbol{E}_{L}^{*}\left(\boldsymbol{k}_{L, 1}, \mathcal{T}_{j-1}^{T} \boldsymbol{r}^{\prime}\right) \cdot \boldsymbol{E}_{L}\left(\boldsymbol{k}_{L, 1}, \mathcal{T}_{l-1}^{T} \boldsymbol{r}^{\prime}\right) d^{2} \boldsymbol{r}^{\prime}
$$

The defect mode in Eq. (38) must have constant phase in space, otherwise net energy transport would occur within the mode. However, the Bloch modes may, under certain circumstances, have simultaneously a real and an imaginary part. Finding a linear combination of Bloch modes that then always sum to give a real, or constant phase, quantity can be complicated, and so details of this process are given in the Appendix. In formulating the combination in the most general way, it occurs that the appropriate partitioning of the Bloch modes is a split into real and imaginary parts, each characterized by a specific symmetry that is dependent on the position of $\boldsymbol{k}_{L, 1}$ within the BZ. The component of the real and imaginary parts of the modes $\psi_{L}\left(\boldsymbol{k}_{L, 1}, \boldsymbol{r}\right)$ can then be expanded in a multipole series:

$\mathcal{F}(\psi)\left(\boldsymbol{k}_{L, 1}, \boldsymbol{r}\right)=\sum_{l=0}^{\infty} b_{\nu l+m_{F}} f_{\nu l+m_{F}}(r)\left\{\begin{array}{l}\cos \left(\left(\nu l+m_{F}\right) \theta\right) \\ \text { or } \\ \sin \left(\left(\nu l+m_{F}\right) \theta\right)\end{array}\right.$

in which $\nu=4,6$ for square and hexagonal lattices respectively. In Eq. (43) the symbol $\mathcal{F}$ can refer to either the real or imaginary functional arguments "Re" and "Im" with the subscript $F$ in $m_{F}$ replaced with corresponding parameters $r$ or $i$, as appropriate. For a real valued Bloch mode there is no solution associated with the imaginary part and so $m_{F}$ is simply replaced by $m$ in this case. The parameters $m_{r}$ and $m_{i}$ characterize the symmetry of the real and imaginary parts and, for the cases of interest here, take values of 0 for rotationally symmetric modes, 1 for modes which are antisymmetric about a nodal line, and $\nu / 2$ for rotationally antisymmetric modes.

With these multipole forms, we may show that the $B_{l, j}$ are real and go on to establish the following relationship which simplifies the summation on the right hand side of Eq. (41)

$$
\begin{aligned}
& \sum_{j=1}^{\nu_{L}} \mathcal{F}\left(\psi_{L}\right)\left(\boldsymbol{k}_{L, 1}, \boldsymbol{\mathcal { T }}_{j-1}^{T} \boldsymbol{r}\right) B_{l, j}= \\
& g_{m_{F}} \nu \mathcal{F}\left(\psi_{L}\right)\left(\boldsymbol{k}_{L, 1}, \boldsymbol{\mathcal { T }}_{l-1}^{T} \boldsymbol{r}\right) \delta \mathcal{E}_{F} .
\end{aligned}
$$

In this equation $\delta \mathcal{E}_{F}\left(=\delta \mathcal{E}_{r}\right.$ or $\left.\delta \mathcal{E}_{i}\right)$ represents the change, caused by the perturbation, to the electric energy of either the real or imaginary part of the Bloch mode:

$$
\delta \mathcal{E}_{F}=\int_{\mathcal{D}_{0}} \delta \varepsilon\left(\boldsymbol{r}^{\prime}\right)\left\|\boldsymbol{E}_{L}^{F}\left(\boldsymbol{k}_{L, 1}, \boldsymbol{r}^{\prime}\right)\right\|^{2} d^{2} \boldsymbol{r}^{\prime} .
$$

Here, $\boldsymbol{E}_{L}^{F}$ denotes the change in energy of the electric fields $\boldsymbol{E}_{L}^{r}$ and $\boldsymbol{E}_{L}^{i}$, associated respectively with $\operatorname{Re} \psi_{L}$ and $\operatorname{Im} \psi_{L}$, while for $m \in\{0, \ldots, \nu-1\}$,

$$
g_{m}= \begin{cases}1, & \text { if } m=0 \text { or } m=\nu / 2 \\ 1 / 2, & \text { otherwise }\end{cases}
$$

The constant $g_{m}$ is a geometrical factor which characterizes the symmetry of the real and imaginary parts of the Bloch modes, the mathematical origin of which appears in the Appendix. Its physical interpretation is as follows: if a given (real or imaginary) part of the Bloch mode is linearly independent from its image under a canonical rotation of $2 \pi / \nu$ (i.e., the smallest rotation that leaves the lattice unchanged), then $g_{m}=1 / 2$; if, however, this field and its rotated image are linearly dependent under such a rotation then $g_{m}=1$.

The relation (44) together with the trigonometric identities (92)-(94), reduce Eq. (41) to

$$
\begin{array}{r}
\sum_{l \in \mathcal{L}} t_{l} \psi_{L}\left(\boldsymbol{k}_{L, 1}, \mathcal{T}_{l-1}^{T} \boldsymbol{r}\right)=-\frac{\omega_{L} C_{L} A_{\mathrm{WSC}} f_{L}}{4 \pi} \times \\
\ln |\delta \omega| \sum_{l \in \mathcal{L}} t_{l}\left[g_{m_{r}} \operatorname{Re}\left(\psi_{L}\right)\left(\boldsymbol{k}_{L, 1}, \mathcal{T}_{l-1}^{T} \boldsymbol{r}\right) \frac{\delta \mathcal{E}_{r}}{\mathcal{E}_{L}}+\right. \\
\left.i g_{m_{i}} \operatorname{Im}\left(\psi_{L}\right)\left(\boldsymbol{k}_{L, 1}, \boldsymbol{\mathcal { T }}_{l-1}^{T} \boldsymbol{r}\right) \frac{\delta \mathcal{E}_{i}}{\mathcal{E}_{L}}\right],
\end{array}
$$

where $f_{L}=\nu_{L} \theta_{L}$. This is the most general form of the expression from which we extract the modal dispersion relations.

When the Bloch mode at the band edge is purely real, the dispersion equation emerges immediately by simply cancelling the representation of the defect mode that appears on both sides of Eq. (47). When the Bloch modes are complex valued at the gap edge, e.g., as may occur at the K-points in hexagonal lattices, we obtain distinct defect modes with two different dispersion equations, but with the same cut-off. In all cases the dispersion relation takes the form

$$
\frac{-1}{\ln |\delta \omega|+O\left((\delta \omega)^{0}\right)}=\frac{s g_{m_{F}}}{\mathcal{S}} \frac{\delta \mathcal{E}_{F}}{\mathcal{E}_{L}},
$$

in which

$$
\mathcal{S}=\frac{4 \pi}{\omega_{L} A_{\mathrm{WSC}}\left|C_{L}\right| f_{L}}=\frac{2}{\omega_{L} N_{L}},
$$

and $s=\operatorname{sign} C_{L}$. Because $\mathcal{S}$ determines by how much the energy of a defect must change to move it a given distance from the band edge, we refer to $\mathcal{S}$ as the defect inertia. 
From Eq. (23), we have $|\delta \omega|=-s \delta \omega$, with this giving the precise interpretation of $|\delta \omega|$ in Eq. (48).

The quantity

$$
\frac{\delta \mathcal{E}_{F}}{\mathcal{E}_{L}}=\frac{\int_{\mathrm{WSC}} \delta \varepsilon(\boldsymbol{r})\left\|\boldsymbol{E}_{L}^{F}\left(\boldsymbol{k}_{L, 1}, \boldsymbol{r}\right)\right\|^{2} d^{2} \boldsymbol{r}}{\int_{\mathrm{WSC}} \varepsilon(\boldsymbol{r})\left\|\boldsymbol{E}_{L}\left(\boldsymbol{k}_{L, 1}, \boldsymbol{r}\right)\right\|^{2} d^{2} \boldsymbol{r}}
$$

denotes the relative change in the energy within the Wigner-Seitz cell caused by the defect.

The defect inertia $\mathcal{S}$ is a positive quantity, with the sign of the prefactor, which includes the sign of the quantity $C_{L}$ retained in $s$. Since $\left|C_{L}\right|$ is related directly to the density of states according to $N_{L}=f_{L}\left|C_{L}\right| A_{\mathrm{WSC}} /(2 \pi)$ [11], we deduce the final form in Eq. (49) for $\mathcal{S}$.

The general form of the modal dispersion relation (48) contains two quantities which can be positive or negative: the change in energy, $\delta \mathcal{E}_{F}$, and the sign $s$ of the band curvature, with $s= \pm 1$ corresponding to a band minimum and a band maximum. In order for $|\delta \omega|$ to remain small as the perturbation vanishes, the product of these two quantities $s \delta \mathcal{E}_{F}$ must be positive. This observation can be used to establish a priori whether the defect state will appear from the upper or the lower band edge: if the change in parameters causes a positive (negative) change in the electric energy $\delta \mathcal{E}_{F}$, then $s$ must also be positive (negative) near the band edge, indicating that the state will originate at the upper (lower) edge of the band gap.

With this in mind, we recast the general result (48) in the exponential form

$$
|\delta \omega|=\mathcal{A} \exp \left(-\frac{\mathcal{S}}{s g_{m} \delta \mathcal{E}_{F} / \mathcal{E}_{L}}\right)
$$

where $\mathcal{A}$ is a constant which, as mentioned earlier, cannot be determined by a first order perturbation analysis. It is important to note that Eq. (51) denotes a single dispersion equation in the case of real valued Bloch modes, and a pair of dispersion equations (each associated with either the real or imaginary parts) if the Bloch mode is complex valued. Eq. (48) is the leading term of an asymptotic approximation that is valid when $|\delta \omega| \rightarrow 0$, where higher order terms (of order $O\left(|\delta \omega|^{0}\right)$ and higher) are neglected. To construct a higher-order approximation, one must include the extended expansion of the MacDonald function in (28), together with the net contributions to the Green's function from the other $\ell \neq L$ bands in Eq. (16), which are also of constant order in $|\delta \omega|$ and have hereto been neglected. We leave this analysis to future work.

\section{Calculations of field profiles}

The treatment leading to Eq. (33) for the mode field is valid for $\boldsymbol{r}, \boldsymbol{r}^{\prime}$ near the defect region $\mathcal{D}_{0}$ where the leading term of Green's function is dominated by $\ln |\delta \omega|$. To approximate the field far from $\mathcal{D}_{0}$ we must include the Green's functions' decay with the distance $\left|\boldsymbol{r}-\boldsymbol{r}^{\prime}\right|$.
In order to deduce this approximation, we choose the diameter of $\mathcal{D}_{0}$ sufficiently small so that

$$
\boldsymbol{r}-\boldsymbol{r}^{\prime} \approx \boldsymbol{r}-\boldsymbol{r}_{0}, \quad \text { if } \boldsymbol{r}^{\prime} \in \mathcal{D}_{0}
$$

where $\boldsymbol{r}_{0}$ is the centre of $\mathcal{D}_{0}$.

For the sake of brevity, we outline the derivation for real valued Bloch modes, for the simple case in which the defect mode in the vicinity of the defect is well approximated by a single Bloch mode rather than by a linear combination, so that $\psi(\boldsymbol{r}) \approx \psi\left(\boldsymbol{k}_{L, 1}, \boldsymbol{r}\right)$. Given the general leading order estimate (27) for the Green's function, Eq. (33) then takes the form

$$
\psi(\boldsymbol{r}) \approx \sum_{j=1}^{\nu_{L}} \psi_{L}\left(\boldsymbol{k}_{L, 1}, \boldsymbol{\mathcal { T }}_{j-1}^{T} \boldsymbol{r}\right) \int_{\mathcal{D}_{0}} h_{j} d^{2} \boldsymbol{r}^{\prime}
$$

where, for both TE and TM polarizations, the function $h_{j}$ is defined as

$$
\begin{aligned}
h_{j}= & \frac{\omega_{L} C_{L} \theta_{L}}{2 \pi \mathcal{E}_{L}} K_{0}\left(b\left|\boldsymbol{\sigma}^{-1}\left(\boldsymbol{r}-\boldsymbol{r}_{0}\right)\right|\right) \\
& \times \delta \varepsilon\left(\boldsymbol{r}^{\prime}\right) \boldsymbol{E}_{L}^{*}\left(\boldsymbol{k}_{L, 1}, \boldsymbol{\mathcal { T }}_{j-1}^{T} \boldsymbol{r}^{\prime}\right) \cdot \boldsymbol{E}_{L}\left(\boldsymbol{k}_{L, 1}, \boldsymbol{r}^{\prime}\right) .
\end{aligned}
$$

Following the field summation formula (44), Eq. (53) can be simplified so that

$\psi(\boldsymbol{r}) \approx \frac{\omega_{L} C_{L} f_{L} g_{m}}{2 \pi} \frac{\delta \mathcal{E}_{r}}{\mathcal{E}_{L}} K_{0}\left(b\left|\boldsymbol{\sigma}^{-1}\left(\boldsymbol{r}-\boldsymbol{r}_{0}\right)\right|\right) \psi_{L}\left(\boldsymbol{k}_{L, 1}, \boldsymbol{r}\right)(55)$

in which the subscript $r$ in $\delta \mathcal{E}_{r}$ makes clear that the calculation is for a real valued mode. From the mode dispersion equation (48), we have

$$
\psi(\boldsymbol{r}) \approx \psi_{L}\left(\boldsymbol{k}_{L, 1}, \boldsymbol{r}\right) \frac{K_{0}\left(b\left|\boldsymbol{\sigma}^{-1}\left(\boldsymbol{r}-\boldsymbol{r}_{0}\right)\right|\right)}{-\frac{\ln |\delta \omega|}{2}-O\left((\delta \omega)^{0}\right)} .
$$

The term $O\left((\delta \omega)^{0}\right)$ is due to $(-\ln \mathcal{A}) / 2$ where $\mathcal{A}$ is the prefactor in Eq. (51). From the small argument asymptotic expansion for $K_{0}(z)$, we can also estimate the quantity $-(\ln |\delta \omega|) / 2-O\left((\delta \omega)^{0}\right)$ as

$$
-\frac{\ln |\delta \omega|}{2}+\frac{\ln \mathcal{A}}{2} \approx K_{0}\left(b r_{\max }\right)
$$

Since we assume that $\psi(\boldsymbol{r}) \approx \psi_{L}\left(\boldsymbol{k}_{L, 1}, \boldsymbol{r}\right)$ for $r$ sufficiently close to the defect, we can approximate $\psi(\boldsymbol{r})$ as a product of the Bloch mode $\psi_{L}\left(\boldsymbol{k}_{L, 1}, \boldsymbol{r}\right)$ by the envelope function

$$
f(\boldsymbol{r})=\left\{\begin{array}{l}
1 \text { if }\left|\boldsymbol{\sigma}^{-1}\left(\boldsymbol{r}-\boldsymbol{r}_{0}\right)\right| \leq r_{\max } \\
\frac{K_{0}\left(b\left|\boldsymbol{\sigma}^{-1}\left(\boldsymbol{r}-\boldsymbol{r}_{0}\right)\right|\right)}{K_{0}\left(b r_{\max }\right)} \text { if }\left|\boldsymbol{\sigma}^{-1}\left(\boldsymbol{r}-\boldsymbol{r}_{0}\right)\right|>r_{\max }
\end{array}\right.
$$

Thus, far from the defect, the defect mode oscillates like the Bloch mode, but within the envelope of a decaying exponential function with decay constant $b$.

Since the quantity $\mathcal{A}$ is not known, we cannot deduce the value of $r_{\max }$ from Eq. (57). However, for the examples considered in this paper, we have good agreement 
between the full numerical calculation and the asymptotic approximation of the field when $r_{\max }$ corresponds to the radius of the defect cylinder or, when $\boldsymbol{\sigma} \neq \boldsymbol{I}$, if the value of $r_{\max }$ is between $a \min \left\{\left|\sigma_{11}\right|^{-1},\left|\sigma_{22}\right|^{-1}\right\}$ and $a \max \left\{\left|\sigma_{11}\right|^{-1},\left|\sigma_{22}\right|^{-1}\right\}$ (we have observed that the best fit occurs for $r_{\max }$ near $\left.a \min \left\{\left|\sigma_{11}\right|^{-1},\left|\sigma_{22}\right|^{-1}\right\}\right)$.

For the example in Fig. 2, we consider the defect created by changing the refractive index of one cylinder from $n_{c}=3$ to $n_{d}=2.5$ in a square lattice of dense cylinders (see also Fig. 6). In Fig. 2 (a), we plot the profile of the defect mode $\psi(\boldsymbol{r})$ near the lower gap edge calculated using the FSS method, while in Fig. 2 (b) we plot the same mode profile using the product of the Bloch mode with the MacDonald function envelope (58). The envelope function is plotted in Fig. 2 (c) while Fig. 2 (d) shows the cross-section of the field profile along the $x$ axis, with there being good agreement between the full numerical calculation (continuous line) and the asymptotic approximation (dashed line). Figs 3 (a) and (b) are the same as Figs 2 (a) and (b), but this time for a defect mode that evolves from the upper gap-edge, with the defect created by changing the refractive index of one cylinder from $n_{c}=3$ to $n_{d}=4$. Figs 3 (c) and (d) show horizontal (through $y=0$ ) and vertical (through $x=a$, $a=0.3 d$ being the cylinder radius) field cross-sections and again there is good agreement between the FSS calculations and the asymptotic theory. Note we set $r_{\max }$ to $a$ and $a \min \left\{\left|\sigma_{11}\right|^{-1},\left|\sigma_{22}\right|^{-1}\right\}$ respectively in Figs 2 and 3 .

\section{Calculations of dispersion relations}

We now investigate several specific instances of defect states evolving from the band edge. The cases that we consider are illustrated in Fig. 4, corresponding to a single cylinder defect created by (a) changing the cylinder refractive index $n_{c}$; (b) changing the cylinder radius $a$; (c) coating a cylinder of refractive index $n_{c}$ and radius $\min \{a, a+\delta a\}$ by a circular shell of refractive index $n_{d}$ and outer radius $\max \{a, a+\delta a\}$; and (d) a change of shape. In each case, we give the specific form of the general asymptotic result (48), viz.

$$
|\delta \omega|=A_{p} \exp \left(-\frac{S_{p}}{s g_{m} \delta p / p}\right)
$$

corresponding to a change in some specific parameter $p$. The quantity $S_{p}$ can be calculated using the Bloch modes at the band edge. These fields may be determined to high accuracy using either the multipole method, as described in Ref. [11], or other techniques, including the finite element method [14], which we have used here. We also simulate the defect state numerically using the Fictitious Source Superposition (FSS) method [12, 13], which is designed explicitly to compute localized states in an infinite array. Using the simulation data, we have generated both a two parameter fit to the model (59) for $S_{p}$ and $A_{p}$, and also a single parameter fit for $A_{p}$, using for $S_{p}$ the value (a)

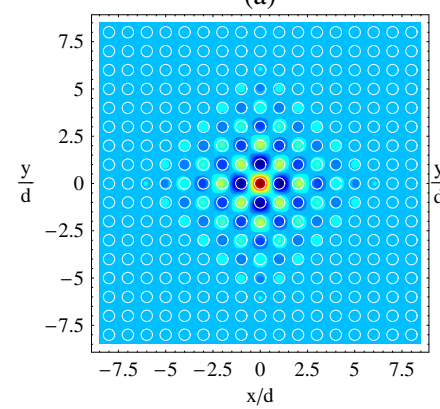

(c)

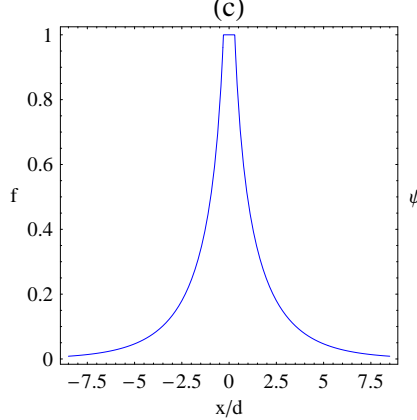

(b)

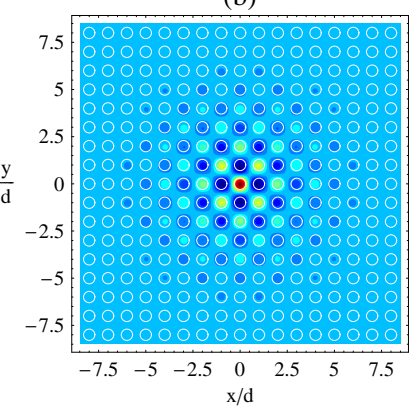

(d)

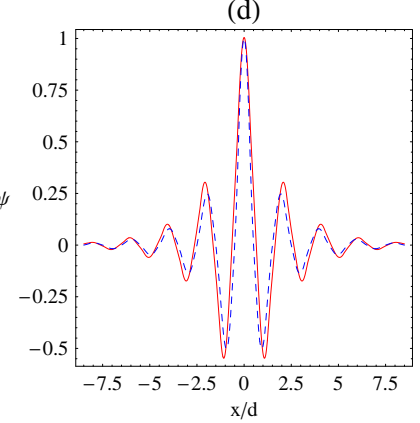

FIG. 2: (Color online) Profile of the defect mode near the lower gap edge: (a) profile calculated using FSS method; (b) profile given by the product of the Bloch mode $\psi_{L}\left(\boldsymbol{k}_{L, 1}, \boldsymbol{\mathcal { T }}_{j-1}^{T} \boldsymbol{r}\right)$ by the envelope $f(\boldsymbol{r})$ defined by Eq. (58). (c) Plot of the envelope function $f(\boldsymbol{r})$ along the $x$-axis. (d) cross-section of the field given by FSS (solid line) and the envelope approximation (dashed line). (a)

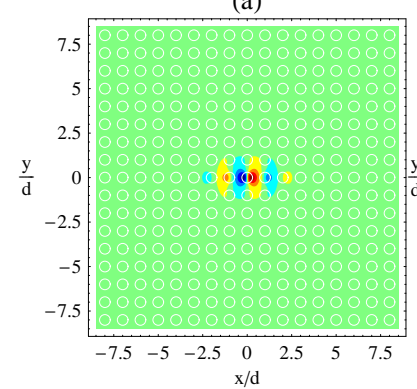

(c)

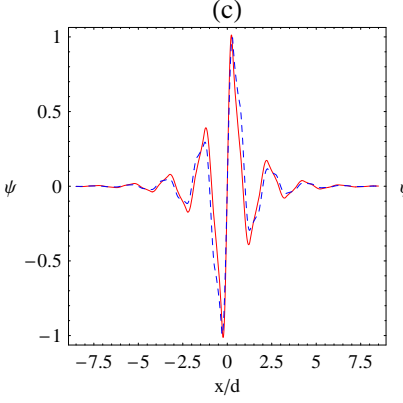

(b)

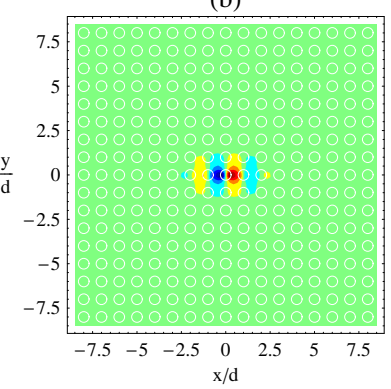

(d)

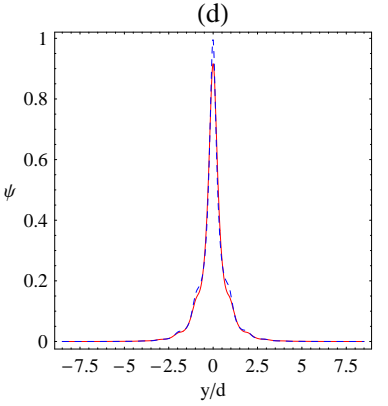

FIG. 3: (Color online) Profile of the defect mode near the upper gap edge: Panels (a) and (b) show modes obtained using respectively the FSS method and the asymptotic formula. Panels (c) and (d) give the cross-section along the directions $y=0$ and $x=a$ where $a=0.3 d$ is the cylinder radius. 
(a)

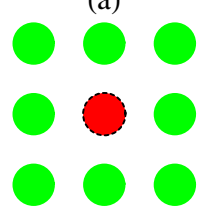

(c)

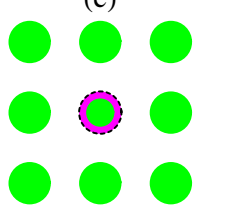

(b)

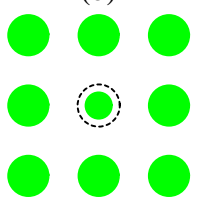

(d)

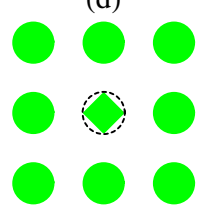

FIG. 4: (Color online) Types of defect considered. Defect created by (a) changing the refractive index $n_{c}$; (b) changing the radius $a$; (c) coating a cylinder; (d) changing its shape. The dashed circle shows the boundary of the unperturbed cylinder.

from the asymptotic theory. These are summarized in Table I, and in all cases the fitted values for $S_{p}$ closely match the values computed using our asymptotic theory. The mode dispersion spectra that follow show both the FSS simulation data and the asymptotic form Eq. (59), with $S_{p}$ computed from the asymptotic analysis and with $A_{p}$ taken from the single parameter fit.

\section{Change of Dielectric Constant}

We consider a general array of cylinders of refractive index $n_{c}$ in a background of refractive index $n_{b}$. For a defect created by changing the refractive index of a single cylinder $C_{0}$ to $n_{d}$, we have

$$
\begin{aligned}
\frac{\delta \mathcal{E}_{F}}{\mathcal{E}_{L}} & =\frac{\int_{D_{0}} \delta \varepsilon(\boldsymbol{r})\left\|\boldsymbol{E}_{L}^{F}\left(\boldsymbol{k}_{L, 1}, \boldsymbol{r}\right)\right\|^{2} d^{2} \boldsymbol{r}}{\int_{\mathrm{WSC}} \varepsilon(\boldsymbol{r})\left\|\boldsymbol{E}_{L}\left(\boldsymbol{k}_{L, 1}, \boldsymbol{r}\right)\right\|^{2} d^{2} \boldsymbol{r}} \\
& =\frac{\delta \varepsilon_{c}}{\varepsilon_{c}} \frac{\int_{D_{0}} \varepsilon_{c}\left\|\boldsymbol{E}_{L}^{F}\left(\boldsymbol{k}_{L, 1}, \boldsymbol{r}\right)\right\|^{2} d^{2} \boldsymbol{r}}{\int_{\mathrm{WSC}} \varepsilon(\boldsymbol{r})\left\|\boldsymbol{E}_{L}\left(\boldsymbol{k}_{L, 1}, \boldsymbol{r}\right)\right\|^{2} d^{2} \boldsymbol{r}},
\end{aligned}
$$

with $\varepsilon_{c}=n_{c}^{2}$ and $\delta \varepsilon_{c}=n_{d}^{2}-n_{c}^{2}$. The change in frequency is then

$$
|\delta \omega|=A_{\delta \varepsilon} \exp \left(-\frac{S_{\delta \varepsilon}}{s g_{m} \delta \varepsilon_{c} / \varepsilon_{c}}\right),
$$

where the value of $S_{\delta \varepsilon}$ is given by

$$
S_{\delta \varepsilon}=\mathcal{S} \frac{\int_{\mathrm{WSC}} \varepsilon(\boldsymbol{r})\left\|\boldsymbol{E}_{L}^{F}\left(\boldsymbol{k}_{L, 1}, \boldsymbol{r}\right)\right\|^{2} d^{2} \boldsymbol{r}}{\int_{D_{0}} \varepsilon_{c}\left\|\boldsymbol{E}_{L}\left(\boldsymbol{k}_{L, 1}, \boldsymbol{r}\right)\right\|^{2} d^{2} \boldsymbol{r}} .
$$

For the first example, we consider a square array of rods with $n_{b}=1, n_{c}=3$, and $a / d=0.3$. For $\mathrm{TM}$ polarization, this $\mathrm{PC}$ has its first band gap for $d / \lambda \in$ $[0.265263,0.334947]$ and, as shown in Fig. 5(a), the lower

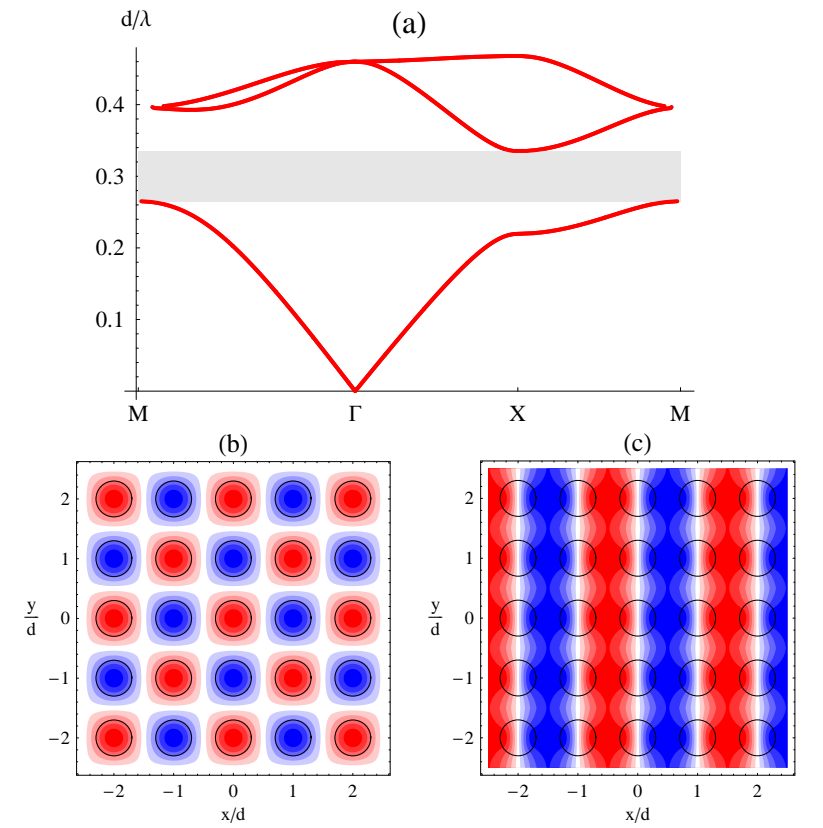

FIG. 5: (Color online) (a) Band diagram for the PC considered in Section IV D 1. (b) Bloch modes at the lower edge ( $M$ point) and (c) upper edge ( $X$ point) of the first band gap. The modes can be normalized so they are purely real.

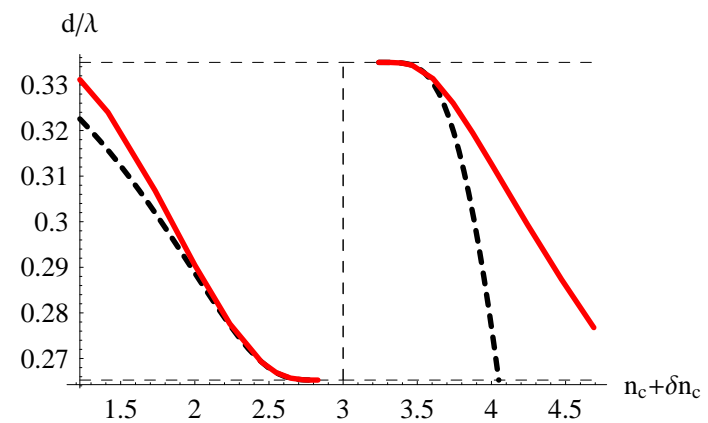

FIG. 6: (Color online) Defect mode evolution in the first gap for the square lattice in Section IV D 1 by changing the refractive index of one cylinder. The red curve shows the defect mode frequency from the FSS method, while the dashed black curves give analytic predictions. The vertical dashed line corresponds to the perfect lattice; the horizontal dashed lines are the gap edges.

and upper gap edges occur respectively at $M$ and $X$ in the BZ. The fields of the Bloch modes at $M$ and $X$ are needed to calculate the parameter $S_{\delta \varepsilon}$ in Eq. (62), and since the array is square symmetric, these modes can be normalized so that they are purely real. Figs 5(b) and (c) show the Bloch modes at these critical points. Both here and in the following calculations, we express all results in terms of a normalized frequency $d / \lambda$ (where $\lambda$ is the free space wavelength, and $d$ is the lattice constant) and a normalized Bloch wavevector $\mathbf{k} d$. 


\begin{tabular}{c|ccccc|} 
Defect type & Edge & $S_{p}$ & $S_{p}^{(2)}$ & $A_{p}$ & $A_{p}^{(2)}$ \\
\hline$\delta \varepsilon(<0)$ & Lower & 1.485 & 1.486 & 0.340 & 0.341 \\
$\delta \varepsilon(>0)$ & Upper & 2.645 & 2.635 & 1.739 & 1.668 \\
\hline$\delta a(<0)$ & Lower & 1.828 & 1.772 & 3.663 & 2.319 \\
$\delta a(>0)$ & Upper & 1.075 & 1.059 & 1.232 & 1.028 \\
\hline Shell $(\delta a<0)$ & Lower & 0.812 & 0.773 & 1.351 & 0.929 \\
Shell $(\delta a>0)$ & Lower & 1.462 & 1.488 & 0.173 & 0.199 \\
\hline
\end{tabular}

TABLE I: (Color online) Comparison of values of the defect inertia factor given by the asymptotic form $S_{p}$ and by the two parameter fit $S_{p}^{(2)}$ for the defect types considered in Section IV D. Also shown are values for the prefactor computed from the single $A_{p}^{(1)}$ and double $A_{p}^{(2)}$ parameter fits. The column labelled edge denotes the band-edge from which the mode originates.

Fig. 6 shows the evolution of the defect mode as the refractive index of the defect cylinder changes from the value $n_{c}$. From Eq. (48) we see that a defect that causes a positive change in the electric energy $\delta \mathcal{E}_{r}$ originates from the upper gap edge while a negative perturbation gives rise to a defect mode originating from the lower edge. For an increase in refractive index, the relative change in energy is positive, and so the defect mode emerges from the top of the gap at $X$, whereas for a decrease in radius it emerges from the lower edge at $M$. This behaviour can be seen in Fig. 6. It can also be seen that there is good agreement between the analytic and numerical results when the defect strength is sufficiently small. The value of parameter $A_{\delta \varepsilon}$ is given in Table $\mathrm{I}$.

\section{Change of Radius}

We next consider a defect created by changing a single cylinder $D_{0}$ of radius $a$ to a defect cylinder $D_{0}^{\prime}$ of radius $a+\delta a$. The integration domain for the defect area now reduces to a shell of thickness $\delta a$ between $D_{0}^{\prime}$ and $D_{0}$; the change in dielectric constant over the shell is $\delta \varepsilon_{\delta a}=$ $\left(n_{c}^{2}-n_{b}^{2}\right) \times \operatorname{sign}(\delta a)$ and since the small parameter is $\delta a$, we do not require $\left(n_{c}^{2}-n_{b}^{2}\right)$ to be small. The relative change in the electric energy is then

$$
\frac{\delta \mathcal{E}_{F}}{\mathcal{E}_{L}}=\frac{\delta \varepsilon_{\delta a} \int_{0}^{2 \pi} \int_{a}^{a+\delta a}\left\|\boldsymbol{E}_{L}^{F}\left(\boldsymbol{k}_{L, 1} ; r, \theta\right)\right\|^{2} r d r d \theta}{\int_{\mathrm{WSC}} \varepsilon(\boldsymbol{r})\left\|\boldsymbol{E}_{L}\left(\boldsymbol{k}_{L, 1}, \boldsymbol{r}\right)\right\|^{2} d^{2} \boldsymbol{r}} .
$$

To first order in $\delta a$, we have

$$
\frac{\delta \mathcal{E}_{\delta a}}{\mathcal{E}_{L}}=\delta a \frac{a \delta \varepsilon_{\delta a} \int_{0}^{2 \pi}\left\|\boldsymbol{E}_{L}^{F}\left(\boldsymbol{k}_{L, 1} ; a, \theta\right)\right\|^{2} d \theta}{\int_{\mathrm{WSC}} \varepsilon(\boldsymbol{r})\left\|\boldsymbol{E}_{L}\left(\boldsymbol{k}_{L, 1}, \boldsymbol{r}\right)\right\|^{2} d^{2} \boldsymbol{r}} .
$$

Thus, the frequency shift is given by

$$
|\delta \omega|=A_{\delta a} \exp \left(-\frac{S_{\delta a}}{s \delta a / a}\right),
$$

where the value of $S_{\delta a}$ is

$$
S_{\delta a}=\frac{S}{a^{2} \delta \varepsilon_{\delta a}} \frac{\int_{\mathrm{WSC}} \varepsilon(\boldsymbol{r})\left\|\boldsymbol{E}_{L}\left(\boldsymbol{k}_{L, 1}, \boldsymbol{r}\right)\right\|^{2} d^{2} \boldsymbol{r}}{\int_{0}^{2 \pi}\left\|\boldsymbol{E}_{L}^{F}\left(\boldsymbol{k}_{L, 1} ; a, \theta\right)\right\|^{2} d \theta} .
$$

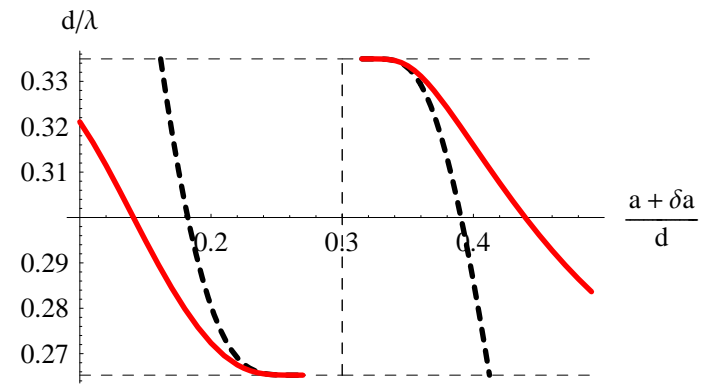

FIG. 7: (Color online) As for Fig. 6 but for a defect formed by changing the radius of one cylinder.

For TE polarization, since $\left\|\nabla \psi_{L}\left(\boldsymbol{k}_{L, 1} ; r, \theta\right)\right\|$ and $\varepsilon(r, \theta)$ are discontinuous across the interface $r=a$, when $\delta a<0$ (resp. $\delta a>0$ ), their values in the region $r<a$ (resp. $r\rangle$ a) should be used to evaluate the electric field intensity $\left\|\boldsymbol{E}_{L}^{F}\left(\boldsymbol{k}_{L, 1} ; a, \theta\right)\right\|^{2}$ which appears in the line integral of Eq. (66).

We consider the same PC as in Section IV D 1, leading to the band diagram and real valued Bloch modes given in Fig. 5. Fig. 7 shows the evolution from the band edge due to the change of radius. For an increase in radius, $\delta \mathcal{E}_{r} / \mathcal{E}_{L}>0$ and so the defect state evolves from the upper band-edge; correspondingly, for a decrease in radius, $\delta \mathcal{E}_{r} / \mathcal{E}_{L}<0$ and so the state emerges from the lower band-edge.

\section{Coating with a circular shell}

Relations (65)-(66) also apply to modes resulting from a defect introduced by coating a cylinder of refractive index $n_{c}$ and radius $\min \{a, a+\delta a\}$ by a circular shell of refractive index $n_{d}$ and outer radius $\max \{a, a+\delta a\}$. By allowing $\delta a$ to be negative, we can study the related situation when the coating of thickness $\delta a$ grows into the cylinder, leaving the outer radius unchanged. The change in dielectric constant over the shell is then $\delta \varepsilon_{\delta a}=$ $\left(n_{d}^{2}-n_{b}^{2}\right)$ when $\delta a>0$ and $\delta \varepsilon_{\delta a}=\left(n_{d}^{2}-n_{c}^{2}\right)$ when $\delta a<0$.

We first consider the square array from Section IV D 1 , where the coating consists of an ideal (i.e., lossless) metal of refractive index $n_{d}=3 i$. Fig. 8 shows the evolution of the defect state from the band-gap edge. For $\delta a>0$, we have $n_{d}^{2}=-9<0$, leading to $\delta \varepsilon<0$ and $\delta \mathcal{E}<0$, and so the defect state evolves from the lower band edge. For $\delta a<0$, the outer radius remains constant and $\delta \varepsilon<0$, giving a net negative energy perturbation, and so the defect state for this perturbation again emerges from the lower band edge.

This type of perturbation can be made very strong, and so is helpful in understanding the role played by the symmetry of the Bloch modes in the creation of defect states. As mentioned previously, in the discussion following equation (42), if the Bloch modes cannot be nor- 
$\mathrm{d} / \lambda$

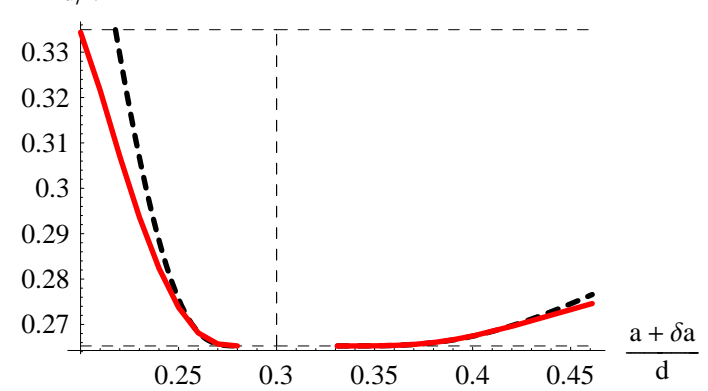

FIG. 8: (Color online) Evolution of the defect mode in the first gap with for the square lattice in Fig. 5 by coating a cylinder with a perfect metal with $n_{d}=3 i$.

malized to purely real quantities then one obtains a pair of dispersion relations corresponding to the symmetric and anti-symmetric (or real and imaginary) parts of $\psi_{L}$. This situation can occur at the $K$ point of the BZ of a hexagonal array, and results in a pair of closely spaced dispersion relations that possess the same cut-off point. Doubly-degenerate defect modes with different dispersion and the same cut-off have been observed before (for example in $[19,20])$, however such "splitting" usually occurs in the context of an asymmetric perturbation. In this case we see that two distinct defect modes arise even when the perturbation is symmetric.

To investigate this we consider a PC with a hexagonal array of holes operated in TE polarization. The holes have refractive index $n_{c}=1$ and normalized radius $a / d=0.3$, and the background has refractive index $n_{b}=3$. As shown in Fig. 9(a), the structure has a band gap for $d / \lambda \in[0.23849,0.30719]$, the lower edge of which occurs at $K$. The real and imaginary parts of the Bloch modes at $K$ are shown in Figs. 9(b) and (c). Note here that the Bloch mode has simultaneously a real and an imaginary part, and that the mode can be normalized so that these parts correspond to the rotationally symmetric and antisymmetric parts of the Bloch mode.

We now consider the defect created by growing a circular shell of refractive index $n_{d}=3 i$, of inner radius $a$, outer radius $a+\delta a$ on one of the cylinders. We consider only $\delta a>0$ because the frequency shift of the defect states is extremely small when the shell is entirely inside a cylinder hole, due to the low electric energy density within the cylinders [10]. Figure 10 shows the evolution of the defect states. As expected, there are two distinct defect mode dispersion curves. The mode profile on the curve containing the point marked "B" closely resembles the product of the real (rotationally symmetric) part of the lower gap edge Bloch mode (see Fig 9(b)) and an envelope function. The profile of the mode shown in the other curve "C" is associated with the imaginary (rotationally antisymmetric) part of the same Bloch mode (Fig 9(c)). Comparing Figs. 9(b) and (c), we see that there is more energy associated with the rotationally

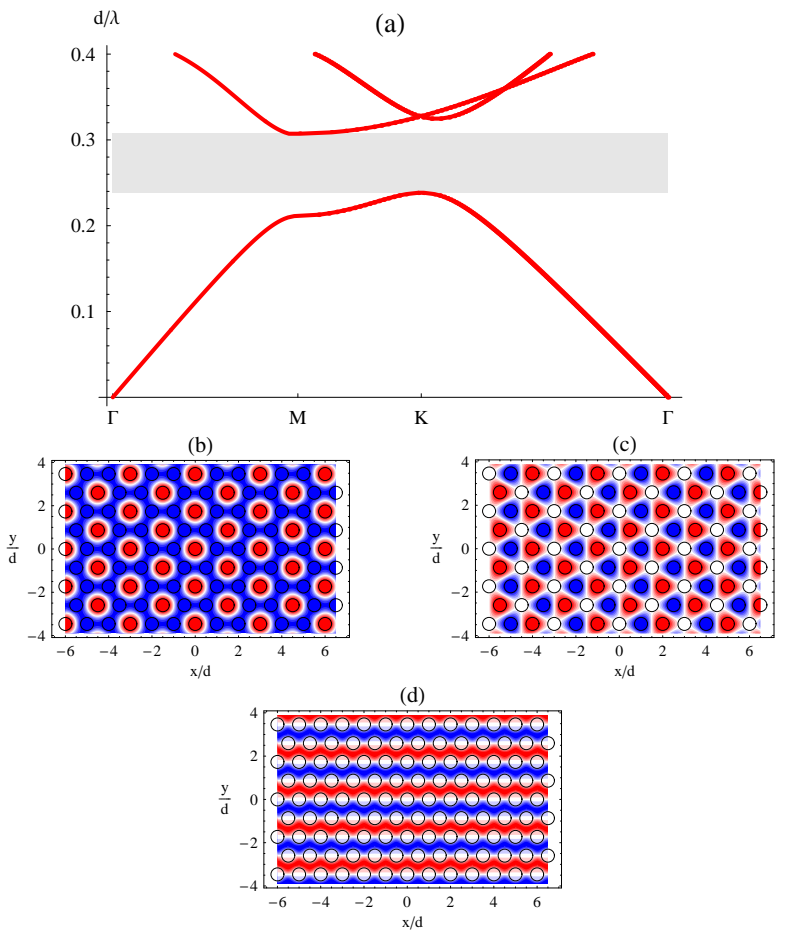

FIG. 9: (Color online) (a) Band diagram for TE polarization in the hexagonal lattice given in Section IV D 3. Panels (b) and (c) present respectively the real part and imaginary part of the Bloch mode at the lower edge ( $K$ point) of the first band gap while the panel $(d)$ shows that at the upper edge ( $M$ point).

symmetric (real) part of the Bloch mode than with the rotationally antisymmetric (imaginary) part, and so the change in the defect frequency caused by the coating will be greater in the case of the former than for the latter. This can be observed in Fig. 10(a), which shows the two dispersion curves for a defect emerging from the $\mathrm{K}$ point of the BZ.

\section{Change of Shape for a Dielectric Cylinder}

We next consider the change of shape of a dielectric cylinder of permittivity $\varepsilon_{c}$ in a background of permittivity $\varepsilon_{b}$. The perturbed boundary of the defect inclusion is described in terms of the small parameter $\eta>0$ as

$$
r(\theta)=a+\eta h(\theta)
$$

where $a$ is the radius of the unperturbed cylinder and $h(\theta)$ is a piecewise smooth function of order $O\left(\eta^{0}\right)$. Without loss of generality, we assume that the ray $\theta=0$ lies on one of the symmetry axes of the Bloch mode $\psi_{L}\left(k_{L}, \mathbf{r}\right)$. The overlap integral extends over the deformed region, with the change in dielectric constant in this region being $\delta \varepsilon(\theta)=\left(\varepsilon_{c}-\varepsilon_{b}\right) \times \operatorname{sign} h(\theta)$. The relative change in 
(a)

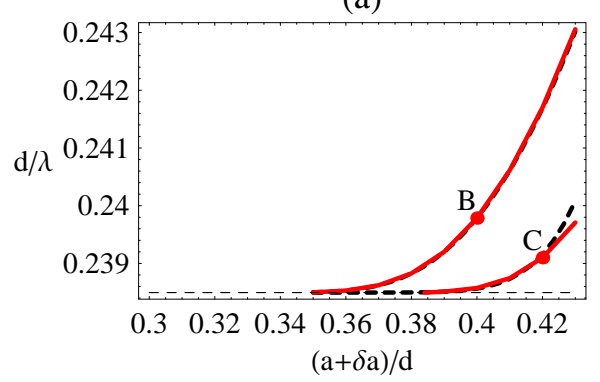

(b)

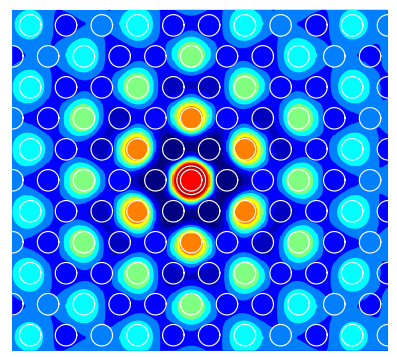

(c)

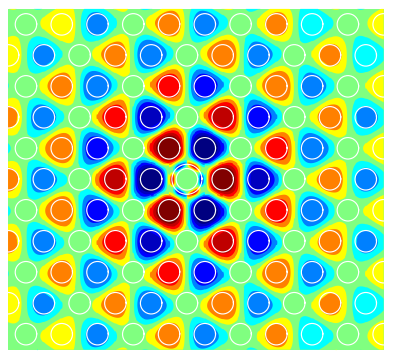

FIG. 10: (Color online) (a) Evolution of the two defect states created by placing a circular shell of refractive index $n_{d}=3 i$, of inner radius $a$ outer radius $a+\delta a$. (b) and (c): Defect modes associated respectively to the points $B$, and $C$ in part (a).

energy is thus

$$
\frac{\delta \mathcal{E}_{F}}{\mathcal{E}_{L}}=\frac{\int_{0}^{2 \pi} \int_{a}^{a+\eta h(\theta)} \delta \varepsilon(\theta)\left\|\boldsymbol{E}_{L}^{F}\left(\boldsymbol{k}_{L, 1} ; r, \theta\right)\right\|^{2} r d r d \theta}{\int_{\mathrm{WSC}} \varepsilon(\boldsymbol{r})\left\|\boldsymbol{E}_{L}\left(\boldsymbol{k}_{L, 1} ; \boldsymbol{r}\right)\right\|^{2} d^{2} \boldsymbol{r}}
$$

Provided that the perturbation region is not too large, we can approximate the integral over this domain using the values of the Bloch functions on the boundary of the unperturbed cylinder, and so

$$
\frac{\delta \mathcal{E}_{F}}{\mathcal{E}_{L}}=\eta \frac{a \int_{0}^{2 \pi} \delta \varepsilon(\theta)|h(\theta)|\left\|\boldsymbol{E}_{L}^{F}\left(\boldsymbol{k}_{L, 1} ; a, \theta\right)\right\|^{2} d \theta}{\int_{\mathrm{WSC}} \varepsilon(\mathbf{r})\left\|\boldsymbol{E}_{L}\left(\boldsymbol{k}_{L, 1} ; r, \theta\right)\right\|^{2} d^{2} \mathbf{r}}+O\left(\eta^{2}\right)
$$

Thus, if $\delta \varepsilon(\theta)$ is an odd function (i.e., if $h(\theta)$ is odd), the effect of the perturbation is of order $O\left(\eta^{2}\right)$. Our asymptotic analysis does not apply to such degenerate perturbations, as may occur with a simple displacement of the cylinder along one of the symmetry axes of the lattice.

The frequency shift for nondegenerate perturbations is

$$
|\delta \omega|=A_{\eta} \exp \left(-S_{\eta} / \eta\right)
$$

where the value of $S_{\eta}$ is

$$
S_{\eta}=\frac{\mathcal{S}}{a} \frac{\int_{\mathrm{WSC}} \varepsilon(\mathbf{r})\left\|\boldsymbol{E}_{L}\left(\boldsymbol{k}_{L, 1} ; r, \theta\right)\right\|^{2} d^{2} \mathbf{r}}{\int_{0}^{2 \pi} \delta \varepsilon(\theta)|h(\theta)|\left\|\boldsymbol{E}_{L}^{F}\left(\boldsymbol{k}_{L, 1} ; a, \theta\right)\right\|^{2} d \theta} .
$$

In Eq. (71), for TE polarization, the intensity $\left\|\boldsymbol{E}_{L}^{F}\left(\boldsymbol{k}_{L, 1} ; r, \theta\right)\right\|^{2}$ is discontinuous across the interface $r=a$ and, as discussed in subsection IV D 3, the sign of $h(\theta)$ will determine which value to use in evaluating the integral over the boundary $r=a$.

For most types of shape perturbation, the defect inertia $S_{\eta}$ is very large because the integral in the denominator is small. This means that the defect state stays close to the band edge even for quite large perturbations, leading to modes that extend large distances from the defect. Such modes cannot be studied by standard numerical algorithms (such as the finite element method) because the computational domain becomes too large. Moreover, the FSS method is presently not suited to the calculation of defect of non-circular inclusions. Accordingly, we have included the asymptotic formula here for completeness, but leave the computations to future work.

\section{Accuracy of the asymptotic formulation: the general model}

The FSS method, which we have used as a standard against which to measure the asymptotic model, is unique in that it can model defects with genuinely infinite claddings [12]. The method uses fictitious sources to tailor the defect mode field and constructs the defect mode from a superposition of quasiperiodic field problems. The superposition requires a $2 \mathrm{D}$ Brillouin zone integration which can be reduced to a $1 \mathrm{D}$ integral by treating the structure as a grating sandwiched between semi-infinite PC mirrors. While the formal theory handles an infinite cladding, the numerical implementation in which the superposition integral is discretized leads to a supercell model, albeit one in which the supercell can be made arbitrarily large - with the accuracy limited only by the number of points with which it discretizes the $1 \mathrm{D}$ Brillouin zone [12]. This choice corresponds to the use of a supercell that is much larger than can be modelled by other numerical means, such as by finite difference time domain or finite element methods. For a sufficiently fine discretization, the FSS method is limited only by machine precision and the results it produces can be considered to be exact, at least from the point of view of comparison with a first-order asymptotic theory. The validity of the asymptotic model can then be quantified by comparison of the values of $S_{p}$ calculated using Eq. (62) and following, with those resulting from a numerical fit to the data using Eq. (59). The results for all defects studied here are collated in Table I, with good agreement (generally within $5 \%$ in all cases) between the computed and fitted values of the defect inertia.

The asymptotic model presented here is a first-order theory, and so can only be expected to be valid in regions near the gap-edge; the calculation of higher-order terms would be necessary to extend the region of applicability deeper into the band gap. The dispersion curves presented in Section IV D are in parametric form, i.e., in terms of independent variables such as the dielectric constant or the radius of the cylinder defect. However, this 
representation does not compare "like with like" and may lead to misinterpretations about the accuracy of the gap edge asymptotic method. For example, in Figs 6 and 7 we see that the approximation departs from the FSS results much more rapidly than in Fig. 8, erroneously suggesting that in the former situations the method is less accurate than in the latter. This, however, is an artefact of the representation of the data; in order to display the data in a uniform and consistent manner, it would be necessary to replot the evolution of the numerically calculated defect modes in terms of their natural variable, namely the relative change in energy $\delta \mathcal{E}_{F} / \mathcal{E}_{L}$. In this form, it would be evident that in the case of high defect inertia (i.e., low density of states), such modes are strongly bound to the band gap, with the FSS and analytic curves coinciding over a wide range of relative energy values. Conversely, for low defect inertia, the FSS and asymptotic curves depart appear to depart rapidly from one another as the mode traverses into the band gap.

For the sake of brevity, we have chosen not to replot all of the results of Section IV D in this form but, instead, to demonstrate the universal nature of the general asymptotic formula (48) by plotting the evolution of the numerically calculated mode frequencies versus the relative change in energy. We would expect that this expression is valid for all calculations for a given lattice type and that, as the defect strength decreases, $-1 / \ln |\delta(d / \lambda)|$ approaches a straight line with slope equal to the defect inertia $\mathcal{S}$. In Fig. 11 we plot $-1 / \ln |\delta(d / \lambda)|$ against the relative change in energy for all square lattice calculations in the previous section and see that the slope approaches $1 / \mathcal{S}$ in each case, confirming the generality of expression (48). We note that computing results for very weak perturbations is very difficult since $-1 / \ln |\delta(d / \lambda)|$ approaches zero extremely slowly as $d / \lambda \rightarrow 0$, e.g., to calculate a point on the ordinate axis of less than 0.05 , it is necessary to calculate $\delta(d / \lambda)$ to within an accuracy of $2 \times 10^{-9}$.

\section{DISCUSSION AND CONCLUSION}

We have presented a substantial analytical treatment, based on first order perturbation theory, explaining the evolution of defect modes from the band gap for a single isolated defect. While the method builds on our earlier work [10] and, in turn, on ideas presented by Economou [21], the extended analysis and the broad range of examples presented here provide a comprehensive treatment of the problem for two-dimensional photonic crystals. While Economou's treatment, in the context of quantum mechanics, uses a tight binding approach to solve Schrödinger's equation, our theory for the Helmholtz equation with polarization dependent boundary conditions makes no such assumptions. We note that result (51), which relates the frequency of the defect state to the density of states at the band edge, a mode symmetry factor and the relative change in the electrical energy

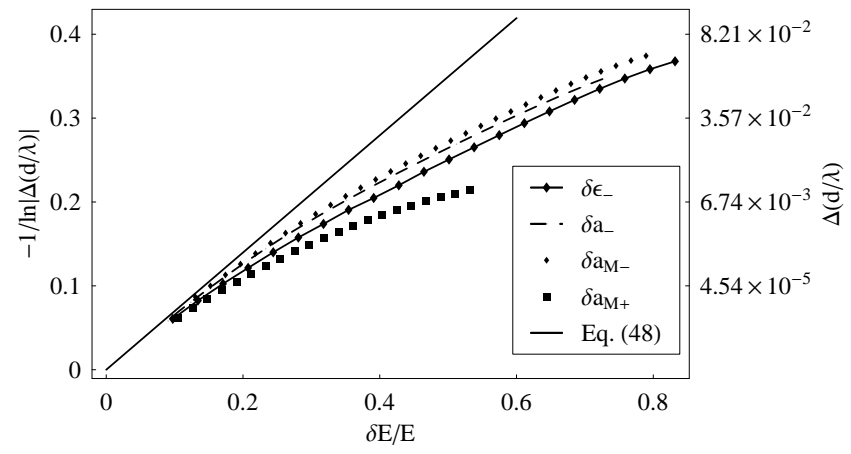

FIG. 11: Evolution of the defect modes for a square array that originate from the lower gap edge versus the relative energy change $\delta \mathcal{E} / \mathcal{E}_{L}$. The legend symbols $\delta \varepsilon_{-}, \delta a_{-}, \delta a_{M-}, \delta a_{M+}$ represent respectively a defect with a change of dielectric constant $\delta \varepsilon<0$, a change of radius $\delta a<0$, and the two cases of metallic skin for $\delta a<0$ and $\delta a>0$. The curve labelled "Eq. (48)" represents $s g_{m} \delta \mathcal{E} /\left(\mathcal{S} \mathcal{E}_{L}\right)$ from Eq. (48).

caused by the introduction of the defect, is remarkably general and can be applied to an arbitrary $2 \mathrm{D}$ photonic crystal geometry. We have demonstrated the validity of this result with a number of numerical calculations, all of which necessitated computations of extreme accuracy, since the theory is only applicable in the vicinity of a band-edge.

An important feature of the results presented here is the defining role played by Bloch mode symmetry in the evolution of defect modes. The situation is relatively straightforward for Bloch modes that are real at the band edge; in this case the defect mode is nondegenerate or two-fold degenerate, depending on the degeneracy of the underlying Bloch function. This manifests itself most notably through the mode symmetry parameter $g_{m}$ (Eq. (46)), the effect of which is to share the change in the electromagnetic energy in the general mode dispersion equation (48) equally between the members of the degenerate pair of Bloch modes. The situation becomes more interesting when the Bloch modes are complex valued and occur in conjugate pairs. In this case, two distinct defect modes arise, possessing the same cutoff but with different dispersion relations, associated, respectively, with the real (or rotationally symmetric) part and the imaginary (or rotationally antisymmetric) part of the Bloch mode. Although such behaviour has been observed for asymmetric perturbations to the material properties $[19,20]$, defect mode splitting that results solely from the asymmetry of the Bloch mode has, to our knowledge, not previously been reported. An analogue exists, however, with the theory of modes in a fibre or waveguide, which can also be non-degenerate, doubly degenerate, or have the same cutoff but differ otherwise. For a guided wave structure, the independent variable is naturally the frequency, whereas for the defect structure it is the defect strength.

We note finally that though the technique we have de- 
veloped is perfectly general, here we have applied it only to fundamental defect modes, which move away from the band edge very slowly with increasing defect strength, and which can be described as being exponentially weakly bound to the band edge. However, when the defect becomes sufficiently strong one can expect higher order defect states to appear. We leave the study of these states to future work.

\section{Acknowledgements}

This work was produced with assistance from the Australian Research Council Centres of Excellence Program.

\section{APPENDIX}

Here, we detail the derivations that lead to the mode dispersion relation that was outlined in Section IV B.

\section{A. Real and complex Bloch modes}

Within a complete band gap, the Green's function $G\left(\boldsymbol{r}, \boldsymbol{r}^{\prime} ; \omega\right)$ and the defect mode $\psi$ must be real valued functions and so we require that their approximation by Bloch mode expansions must also be real valued.

A Bloch mode $\psi_{L}(\boldsymbol{k}, \boldsymbol{r})$ is a solution of the Helmholtz equation that satisfies the quasi-periodic conditions $\psi_{L}\left(\boldsymbol{k}, \boldsymbol{r}+\boldsymbol{e}_{1}\right)=e^{i \boldsymbol{k} \cdot \boldsymbol{e}_{1}} \psi_{L}(\boldsymbol{k}, \boldsymbol{r})$ and $\psi_{L}\left(\boldsymbol{k}, \boldsymbol{r}+\boldsymbol{e}_{2}\right)=$ $e^{i \boldsymbol{k} \cdot \boldsymbol{e}_{2}} \psi_{L}(\boldsymbol{k}, \boldsymbol{r})$ over the boundaries of a parallelogram unit cell generated by the lattice vectors $\boldsymbol{e}_{1}$ and $\boldsymbol{e}_{2}$ (Figs. 12 (a) and (b)). A Bloch mode may be normalized to be a real function if the conjugate mode $\psi_{L}^{*}(\boldsymbol{k}, \boldsymbol{r})$ is also a solution of the same boundary value problem. For this to be the case, the Bloch factors $\mu_{1}=e^{i \boldsymbol{k} \cdot \boldsymbol{e}_{1}}$ and $\mu_{2}=e^{i \boldsymbol{k} \cdot \boldsymbol{e}_{2}}$ must be real, i.e., $\mu_{1}= \pm 1$ and $\mu_{2}= \pm 1$.

From the representation of the Bloch mode $\psi_{L}(\boldsymbol{k}, \boldsymbol{r})$ as the product of a periodic function and a plane wave term $e^{i \boldsymbol{k} \cdot \boldsymbol{r}}$ and also from the symmetry of the lattice, it follows that $\psi_{L}^{*}(\boldsymbol{k}, \boldsymbol{r})$ and $\psi_{L}(\boldsymbol{k},-\boldsymbol{r})$ are Bloch modes associated with Bloch vector $\boldsymbol{- k}$. We may then normalize $\psi_{L}(\boldsymbol{k}, \boldsymbol{r})$ to be real by scaling it to ensure that $\psi_{L}^{*}(\boldsymbol{k}, \boldsymbol{r})=$ $\psi_{L}(\boldsymbol{k},-\boldsymbol{r})$.

For a square lattice, the Bloch factors $\left(e^{i \boldsymbol{k} \cdot \boldsymbol{e}_{l}}\right)$ are real numbers when $\boldsymbol{k}$ is associated with any one of the symmetry points $\Gamma, M_{j}$ and $X_{j}$ shown in Fig. 12(c). In contrast, for hexagonal lattices the Bloch factors are real for $\boldsymbol{k}$ at $\Gamma$ or $M_{j}$, but not at $K_{j}$. For example, if $\boldsymbol{k}$ corresponds to $K_{1}$, then $\boldsymbol{k} \cdot \boldsymbol{e}_{1}=2 \pi / 3$ [22] and so $\mu_{1}$ is complex. Thus, for a hexagonal lattice the Bloch modes can be taken as real valued at $\Gamma$ and $M_{j}$, but not at the $K_{j}$. To understand how to deal with the $K_{j}$ points, since we need a real valued Bloch mode series representation of the defect mode, we consider the situation in Fig. 12(d) and demonstrate that there are only two linearly independent states. To see this, the six $K$ points are partitioned into two sets $\left\{K_{1}, K_{3}, K_{5}\right\}$ and $\left\{K_{2}, K_{4}, K_{6}\right\}$. The points $K_{3}$ and $K_{5}$ respectively differ from $K_{1}$ by reciprocal lattice vectors $\boldsymbol{u}_{1}$ and $\boldsymbol{u}_{2}$ (see Fig. 12(d)) and so correspond to the same Bloch mode. Similarly, $\left\{K_{2}, K_{4}, K_{6}\right\}$ also correspond to the same mode. We denote the first mode by $\psi_{L}\left(\boldsymbol{k}_{L, 1}, \boldsymbol{r}\right)$ and deduce the second member of the linearly independent pair by noting that $\boldsymbol{k}_{L, 4}=-\boldsymbol{k}_{L, 1}$. From this it follows that $\psi_{L}\left(\boldsymbol{k}_{L, 4}, \boldsymbol{r}\right)=\psi_{L}\left(\boldsymbol{k}_{L, 1},-\boldsymbol{r}\right)$ which, in turn, is associated with $\psi_{L}^{*}\left(\boldsymbol{k}_{L, 1}, \boldsymbol{r}\right)$. We thus normalize the pair of modes by ensuring that $\psi_{L}\left(\boldsymbol{k}_{L, 1},-\boldsymbol{r}\right)=\psi_{L}^{*}\left(\boldsymbol{k}_{L, 1}, \boldsymbol{r}\right)$; in particular, this implies that the real part of $\psi_{L}\left(\boldsymbol{k}_{L, 1}, \boldsymbol{r}\right)$ has even symmetry, with respect to $\boldsymbol{r}=\mathbf{0}$, while the imaginary part is odd. Thus, the set of Bloch modes at the gap edge is always composed of either real modes or conjugate pairs of complex valued modes.

\section{B. Mode symmetry and multipole representations}

To proceed with the derivation of explicit forms, we introduce a formalism that encapsulates symmetry in a convenient mathematical notation. We do this with a general multipole representation, expressing the field in the Wigner-Seitz cell as

$$
\psi_{L}\left(\boldsymbol{k}_{L, 1}, \boldsymbol{r}\right)=\sum_{l=-\infty}^{\infty} \tilde{b}_{l} f_{l}(r) \exp (i l \theta) .
$$

In Eq. (72), the $\left\{f_{n}(r)\right\}$ are expressed in terms of cylindrical harmonic functions. Within the cylinders, the $\left\{f_{n}(r)\right\}$ are expanded in regular Bessel functions of the first kind $\left(J_{n}\right)$ while outside the cylinders they are expanded as a linear combination of Bessel functions $\left(J_{n}\right)$ and Hankel functions of the first kind $\left(H_{n}^{(1)}\right)$. Below, the precise form of the $\left\{f_{n}(r)\right\}$ is irrelevant; only the symmetry of the mode with respect to the azimuthal angle $\theta$ is significant.

If $\psi_{L}\left(\boldsymbol{k}_{L, 1}, \boldsymbol{r}\right)$ is even or odd with respect to the variable $\theta$, the complex exponential representation (72) can be replaced respectively by a cosine or sine series

$$
\psi_{L}\left(\boldsymbol{k}_{L, 1}, \boldsymbol{r}\right)=\sum_{l=0}^{\infty} b_{l} f_{l}(r)\left\{\begin{array}{l}
\cos l \theta \\
\sin l \theta
\end{array}\right.
$$

To enforce the rotational symmetry with respect to the defect centre, only terms with compatible symmetry are retained; thus Eq. (73) becomes

$$
\psi_{L}\left(\boldsymbol{k}_{L, 1}, \boldsymbol{r}\right)=\sum_{l=0}^{\infty} b_{\nu l+m} f_{\nu l+m}(r)\left\{\begin{array}{l}
\cos (\nu l+m) \theta \\
\sin (\nu l+m) \theta
\end{array},\right.
$$

in which $\nu=4,6$ for square, hexagonal lattices, respectively. Only a small number of cases are of interest to us. For square lattices, these are $m=0$ for modes of the bulk PC with rotational symmetry, and $m=1$ for modes which are antisymmetric about a nodal line, while 
for hexagonal lattices these are $m=0$ and $m=1$, as for square lattices, and $m=3$ which we discuss in some detail below.

A Bloch mode $\psi_{L}\left(\boldsymbol{k}_{L, 1}, \boldsymbol{r}\right)$ that is represented by Eq. (73) may be scaled to be real. To see this, note that the eigenfunctions $\psi_{L}^{*}\left(\boldsymbol{k}_{L, 1}, \boldsymbol{r}\right)$ and $\psi_{L}\left(\boldsymbol{k}_{L, 1}, \boldsymbol{\mathcal { T }}_{\nu / 2}^{T} \boldsymbol{r}\right)=$ $\psi_{L}\left(\boldsymbol{k}_{L, 1},-\boldsymbol{r}\right)$ are both associated with the wavevector $-\boldsymbol{k}_{L, 1}$ and so are proportional to one another, i.e., there exists a complex number $\alpha$ such that $\psi_{L}^{*}\left(\boldsymbol{k}_{L, 1}, \boldsymbol{r}\right)=$ $\alpha \psi_{L}\left(\boldsymbol{k}_{L, 1},-\boldsymbol{r}\right)$. The coefficients $b_{\nu l+m}$ in the multipole representation $(74)$ then satisfy the relation $b_{\nu l+m}^{*}=$ $(-1)^{m} \alpha b_{\nu l+m}$, and so $|\alpha|=1$. Now, if $\beta$ is a complex number such that $\left(\beta^{*}\right)^{2}=(-1)^{m} / \alpha$, then $(-1)^{m} \beta^{*} \alpha=$ $1 / \beta^{*}=\beta$ since $\beta \beta^{*}=|\alpha|^{2}=1$. It is then straightforward to show that $\left(\beta b_{\nu l+m}\right)^{*}=\beta b_{\nu l+m}$ which, in turn, allows us to construct a real Bloch mode $\beta \psi_{L}\left(\boldsymbol{k}_{L, 1}, \boldsymbol{r}\right)$, as required.

Figs 5 and 9 show Bloch modes at the band edge for the examples used in this paper. In Fig. 5 for a square lattice, the rotational symmetry of $\psi_{L}(\boldsymbol{k}, \boldsymbol{r})$ at $M_{1}$ is encapsulated by the choice $(\nu, m)=(4,0)$ in the cosine form of multipole representation (74), while the even symmetry of the mode (with respect to the $y$-axis) at $X_{1}$ is represented by the cosine form of the multipole expansion $(74)$ with $(\nu, m)=(4,1)$. For the hexagonal lattice, the odd symmetry of the mode at $M_{1}$ is realized using the sine representation (74) and the choice of parameters $(\nu, m)=(6,1)$. At $K_{1}$, which we denote by $\boldsymbol{k}\left(K_{1}\right)$, the mode $\psi_{L}\left(\boldsymbol{k}\left(K_{1}\right), \boldsymbol{r}\right)$ is complex valued, and attains its maximum magnitude at the centre of each cylinder $(\boldsymbol{r}=\mathbf{0})$. In Fig. 9, $\psi_{L}\left(\boldsymbol{k}\left(K_{1}\right), \boldsymbol{r}\right)$ is scaled to be real at the cylinder centre and the resulting field satisfies $\psi_{L}\left(\boldsymbol{k}\left(K_{1}\right),-\boldsymbol{r}\right)=\psi_{L}^{*}\left(\boldsymbol{k}\left(K_{1}\right), \boldsymbol{r}\right)$. Both real and imaginary parts are even with respect to $\theta$. They also satisfy

$$
\begin{aligned}
& \operatorname{Re} \psi_{L}\left(\boldsymbol{k}\left(K_{1}\right), \boldsymbol{T} \boldsymbol{r}\right)=\operatorname{Re} \psi_{L}\left(\boldsymbol{k}\left(K_{1}\right), \boldsymbol{r}\right), \\
& \operatorname{Im} \psi_{L}\left(\boldsymbol{k}\left(K_{1}\right), \mathcal{T} \boldsymbol{r}\right)=-\operatorname{Im} \psi_{L}\left(\boldsymbol{k}\left(K_{1}\right), \boldsymbol{r}\right),
\end{aligned}
$$

in which $\mathcal{T}$ denotes a canonical rotation of $\pi / 3$. To characterize these symmetry properties, we respectively represent the real and imaginary parts of the mode by the cosine form of expansion (74) using $(\nu, m)=(6,0)$ and $(\nu, m)=(6,3)$.

\section{Key results leading to the mode dispersion equation}

We now derive key results that simplify the summations in Eq. (33), allowing us to find the dispersion equation for the defect mode. Recall that the set of Bloch modes $\mathcal{M}=\left\{\psi_{L}\left(\boldsymbol{k}_{L, 1}, \boldsymbol{\mathcal { T }}_{j-1}^{T} \boldsymbol{r}\right)\right\}$, for $j=1, \ldots, \nu$, is not linearly independent. We thus select from $\mathcal{M}$ a basis $\mathcal{L}$ of linearly independent functions that can generate the members of $\mathcal{M}$. For a square lattice, the four $M_{j}$ (Fig. 12) constitute the simplest case since all are associated with the same real valued Bloch mode. The set $\mathcal{L}$ thus contains only a single function, i.e., $\mathcal{L}=$ (a)
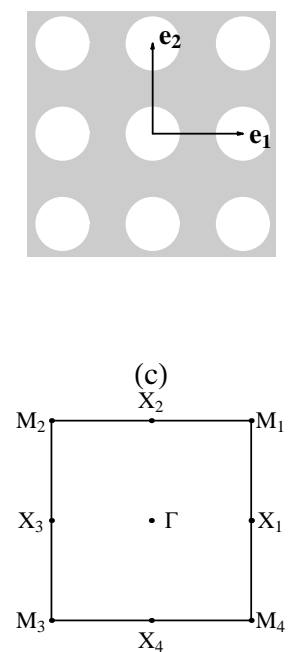

(b)

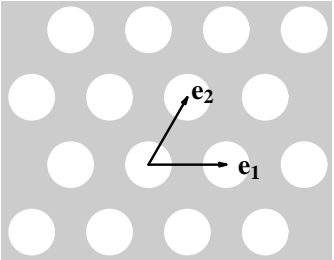

(d)

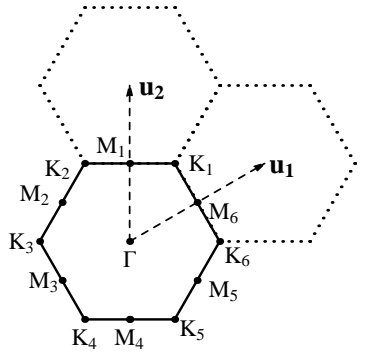

FIG. 12: (a) Basis vectors for a square lattice. (b) Basis vectors for a hexagonal lattice. (c) The first BZ for the square lattice; the four $M_{j}$ points are equivalent Bloch wavevectors; as are $\left(X_{1}, X_{3}\right)$ and $\left(X_{2}, X_{4}\right)$. (d) The first BZ, and two of its replicates in reciprocal space for a hexagonal lattice. $K_{1}, K_{3}$ and $K_{5}$ are equivalent since they differ by reciprocal lattice vectors. Similarly, each member of $\left\{K_{2}, K_{4}, K_{6}\right\},\left\{M_{1}, M_{4}\right\}$, $\left\{M_{2}, M_{5}\right\}$ and $\left\{M_{3}, M_{6}\right\}$ are equivalent.

$\left\{\psi\left(\boldsymbol{k}\left(M_{1}\right), \boldsymbol{r}\right)\right\}$. The four $X_{j}$ have two independent, real valued modes and so $\mathcal{L}=\left\{\psi\left(\boldsymbol{k}\left(X_{1}\right), \boldsymbol{r}\right), \psi\left(\boldsymbol{k}\left(X_{2}\right), \boldsymbol{r}\right)\right\}$. For a hexagonal lattice, the six $M_{j}$ define three different Bloch modes, only two of which are linearly independent. Taking the three modes to be $\psi_{L}\left(\boldsymbol{k}_{L, 1}, \mathcal{T}_{j-1}^{T} \boldsymbol{r}\right)$ for $j=1,2,3$, it is easily shown, using the sine form of expansion (74), that

$$
\psi_{L}\left(\boldsymbol{k}_{L, 1}, \mathcal{T}_{1}^{T} \boldsymbol{r}\right)=\frac{1}{2}\left(\psi_{L}\left(\boldsymbol{k}_{L, 1}, \boldsymbol{r}\right)+\psi_{L}\left(\boldsymbol{k}_{L, 1}, \boldsymbol{\mathcal { T }}_{2}^{T} \boldsymbol{r}\right)\right)
$$

and so the linearly independent set is $\mathcal{L}=$ $\left\{\psi_{L}\left(\boldsymbol{k}_{L, 1}, \boldsymbol{r}\right), \psi_{L}\left(\boldsymbol{k}_{L, 1}, \mathcal{T}_{2}^{T} \boldsymbol{r}\right)\right\}$.

The derivation of the defect mode dispersion relation relies on the multipole representations (74). We further take the defect to be rotationally symmetric, i.e., $\delta \varepsilon(\boldsymbol{r})=\delta \varepsilon(\boldsymbol{T} \boldsymbol{r})$. Recall that the representations (74) are valid only for real Bloch modes, and so some care is required in dealing with the remaining, and most complicated, case that we consider, namely that for the six $K$ points of the hexagonal lattice, at which the mode is complex valued. We take the modes to be normalized such that $\psi_{L}^{*}\left(\boldsymbol{k}_{L, 1}, \boldsymbol{r}\right)=\psi_{L}\left(\boldsymbol{k}_{L, 1},-\boldsymbol{r}\right)$ which, in turn, prescribes the real part of the mode to be symmetric and the imaginary part to be antisymmetric. These may be represented by either the cosine or sine forms of Eq. (74), with the different symmetries (of the real and imaginary parts) simplifying the later analysis since integrals involving their product vanish. 
The derivation of the key relations commences with the expansion of the defect mode $\psi(\boldsymbol{r})$ and the associated electric field $\boldsymbol{E}(\boldsymbol{r})$ in the basis of linearly independent Bloch modes

$$
\begin{aligned}
\psi(\boldsymbol{r}) & \approx \sum_{l \in \mathcal{L}} t_{l} \psi_{L}\left(\boldsymbol{k}_{L, 1}, \mathcal{T}_{l-1}^{T} \boldsymbol{r}\right) \\
\boldsymbol{E}(\boldsymbol{r}) & \approx \sum_{l \in \mathcal{L}} t_{l} \boldsymbol{E}_{L}\left(\boldsymbol{k}_{L, 1}, \mathcal{T}_{l-1}^{T} \boldsymbol{r}\right) .
\end{aligned}
$$

Substitution of the approximations (78) and (79) into Eq. (33) leads to

$$
\begin{aligned}
& \sum_{l \in \mathcal{L}} t_{l} \psi_{L}\left(\boldsymbol{k}_{L, 1}, \mathcal{T}_{l-1}^{T} \boldsymbol{r}\right)=-\frac{\omega_{L} C_{L} A_{\mathrm{WSC}} \theta_{L}}{4 \pi \mathcal{E}_{L}} \\
& \times \ln |\delta \omega| \sum_{l \in \mathcal{L}} t_{l} \sum_{j=1}^{\nu_{L}} B_{l, j} \psi_{L}\left(\boldsymbol{k}_{L, 1}, \mathcal{T}_{j-1}^{T} \boldsymbol{r}\right)
\end{aligned}
$$

in which

$$
B_{l, j}=\int_{\mathcal{D}_{0}} \delta \varepsilon\left(\boldsymbol{r}^{\prime}\right) \boldsymbol{E}_{L}^{*}\left(\boldsymbol{k}_{L, 1}, \mathcal{T}_{j-1}^{T} \boldsymbol{r}^{\prime}\right) \cdot \boldsymbol{E}_{L}\left(\boldsymbol{k}_{L, 1}, \mathcal{T}_{l-1}^{T} \boldsymbol{r}^{\prime}\right) d^{2} \boldsymbol{r}^{\prime}
$$

In evaluating the coefficients $B_{l, j}(81)$, we express the terms $\boldsymbol{E}_{L}\left(\boldsymbol{k}_{L, 1}, \boldsymbol{\mathcal { T }}_{j-1}^{T} \boldsymbol{r}^{\prime}\right)$ in Eq. (81) in terms of the real and imaginary parts of the Bloch mode $\psi_{L}\left(\boldsymbol{k}_{L, 1}, \boldsymbol{\mathcal { T }}_{j-1}^{T} \boldsymbol{r}^{\prime}\right)$ and deduce

$$
B_{l, j}=\left(B_{l, j}^{\mathrm{RR}}+B_{l, j}^{\mathrm{II}}\right)+i\left(B_{l, j}^{\mathrm{RI}}-B_{l, j}^{\mathrm{IR}}\right),
$$

with

$$
\begin{aligned}
B_{l, j}^{\mathrm{FG}}=\int_{\mathcal{D}_{0}} & \delta \\
& \left(\boldsymbol{r}^{\prime}\right) \mathcal{F} \psi_{L}\left(\boldsymbol{k}_{L, 1}, \boldsymbol{\mathcal { T }}_{j-1}^{T} \boldsymbol{r}^{\prime}\right) \\
& \times \mathcal{G} \psi_{L}\left(\boldsymbol{k}_{L, 1}, \boldsymbol{\mathcal { T }}_{l-1}^{T} \boldsymbol{r}^{\prime}\right) d^{2} \boldsymbol{r}^{\prime}
\end{aligned}
$$

for TM polarization and

$$
\begin{array}{r}
B_{l, j}^{\mathrm{FG}}=\int_{\mathcal{D}_{0}} \frac{\delta \varepsilon\left(\boldsymbol{r}^{\prime}\right)}{\omega^{2} \varepsilon\left(\boldsymbol{r}^{\prime}\right)^{2}} \nabla\left[\mathcal{F} \psi_{L}\left(\boldsymbol{k}_{L, 1}, \boldsymbol{\mathcal { T }}_{j-1}^{T} \boldsymbol{r}^{\prime}\right)\right] \\
\cdot \nabla\left[\mathcal{G} \psi_{L}\left(\boldsymbol{k}_{L, 1}, \boldsymbol{\mathcal { T }}_{l-1}^{T} \boldsymbol{r}^{\prime}\right) d^{2} \boldsymbol{r}^{\prime}\right]
\end{array}
$$

and for TE polarization.

In Eqs (83) and (84) the symbols $\mathcal{F}$ and $\mathcal{G}$ may refer to either the real or imaginary functional arguments "Re" and "Im", with the superscripts F and G on the left-hand side replaced by parameters $\mathrm{R}$ or I as appropriate.

The modes are normalized, as described above, using the relation $\psi_{L}^{*}\left(\boldsymbol{k}_{L, 1}, \boldsymbol{r}\right)=\psi_{L}\left(\boldsymbol{k}_{L, 1},-\boldsymbol{r}\right)$. In general, the real and imaginary parts of the mode have different symmetries, and so their respective expansions (74) are characterized by different values of the parameter $m$. We thus introduce $m=m_{r}$ and $m=m_{i}$ respectively for the real and imaginary parts.
With the aid of the multipole representation (74) and the following trigonometric identities (for integer $n$ ),

$$
\begin{gathered}
\int_{0}^{2 \pi} \cos (n \theta) \cos \left(n\left(\theta+\theta_{0}\right)\right) d \theta=\cos \left(n \theta_{0}\right) \int_{0}^{2 \pi} \cos ^{2}(n \theta) d \theta,(85) \\
\int_{0}^{2 \pi} \sin (n \theta) \sin \left(n\left(\theta+\theta_{0}\right)\right) d \theta=\cos \left(n \theta_{0}\right) \int_{0}^{2 \pi} \sin ^{2}(n \theta) d \theta,(86)
\end{gathered}
$$

we may simplify $B_{l, j}^{\mathrm{RR}}$ and $B_{l, j}^{\mathrm{II}}$ as follows

$$
\begin{aligned}
B_{l, j}^{\mathrm{RR}} & =\cos \left(\frac{2 \pi(j-l) m_{r}}{\nu}\right) \delta \mathcal{E}_{\mathrm{R}}, \\
B_{l, j}^{\mathrm{II}} & =\cos \left(\frac{2 \pi(j-l) m_{i}}{\nu}\right) \delta \mathcal{E}_{\mathrm{I}},
\end{aligned}
$$

where $\delta \mathcal{E}_{r}$ and $\delta \mathcal{E}_{i}$ are defined by

$$
\delta \mathcal{E}_{\mathrm{R}}=B_{1,1}^{\mathrm{RR}} \quad \text { and } \quad \delta \mathcal{E}_{\mathrm{I}}=B_{1,1}^{\mathrm{II}} .
$$

Since the real and imaginary parts of the Bloch mode exhibit different symmetries (a consequence of the identity $\left.\psi_{L}\left(\boldsymbol{k}_{L, 1},-\boldsymbol{r}\right)=\psi_{L}^{*}\left(\boldsymbol{k}_{L, 1}, \boldsymbol{r}\right)\right)$, we have $m_{r} \neq m_{i}$ and so

$$
\begin{aligned}
& B_{l, j}^{\mathrm{RI}}=0, \\
& B_{l, j}^{\mathrm{IR}}=0 .
\end{aligned}
$$

Following some straightforward manipulation, together with the application of the trigonometric identities

$$
\begin{aligned}
\frac{1}{\nu} \sum_{j=1}^{\nu} \cos ^{2}\left(\frac{2 \pi(j-1) m}{\nu}\right) & \stackrel{\text { def }}{=} g_{m}, \\
\sum_{j=1}^{\nu} \sin \left(\frac{2 \pi(j-1) m}{\nu}\right) \cos \left(\frac{2 \pi(j-1) m}{\nu}\right) & =0 \\
\sum_{j=1}^{\nu} \cos \left(\frac{2 \pi(j-1) m_{r}}{\nu}\right) \cos \left(\frac{2 \pi(j-1) m_{i}}{\nu}\right) & =0
\end{aligned}
$$

we derive

$$
\begin{aligned}
& \sum_{j=1}^{\nu_{L}}\left(\mathcal{F}\left(\psi_{L}\right)\left(\boldsymbol{k}_{L, 1}, \mathcal{T}_{j-1}^{T} \boldsymbol{r}\right) B_{l, j}^{\mathrm{FF}}\right) \\
& =\nu g_{m_{F}} \mathcal{F}\left(\psi_{L}\right)\left(\boldsymbol{k}_{L, 1}, \boldsymbol{\mathcal { T }}_{l-1}^{T} \boldsymbol{r}\right) \delta \mathcal{E}_{\mathrm{F}}
\end{aligned}
$$

for both the real and imaginary parts (using our abbreviated and general nomenclature) and where, for the integer $m_{F} \in\{0, \ldots, \nu-1\}$,

$$
g_{m_{F}}= \begin{cases}1, & \text { if } m_{F}=0 \text { or } m_{F}=\nu / 2 \\ \frac{1}{2}, & \text { otherwise. }\end{cases}
$$

The quantity $g_{m_{F}}$ characterizes the symmetry of the mode, and, from definition (92) can be thought of as follows: if, under a canonical rotation of $2 \pi / \nu$, the modal quantity is unchanged in the sense that it and the unrotated mode are linearly dependent then $g_{m_{F}}=1$, otherwise $g_{m_{F}}=1 / 2$. 
Relations (95) simplify the summation that appears on the right hand side of Eq. (80) and allow us to find that

$$
\begin{aligned}
& \sum_{l \in \mathcal{L}} t_{l} \psi_{L}\left(\boldsymbol{k}_{L, 1}, \mathcal{T}_{l-1}^{T} \boldsymbol{r}\right)=-\frac{\omega_{L} C_{L} A_{\mathrm{WSC}} f_{L}}{4 \pi} \\
& \times \ln |\delta \omega|\left(g_{m_{r}} \mathcal{S}_{r}+i g_{m_{i}} \mathcal{S}_{i}\right),
\end{aligned}
$$

where $f_{L}=\nu_{L} \theta_{L}$, and

$$
\mathcal{S}_{F}=\frac{\delta \mathcal{E}_{F}}{\mathcal{E}_{L}} \sum_{l \in \mathcal{L}} t_{l} \mathcal{F}\left(\psi_{L}\right)\left(\boldsymbol{k}_{L, 1}, \boldsymbol{\mathcal { T }}_{l-1}^{T} \boldsymbol{r}\right),
$$

in which $\mathcal{F}$ and $F$ may refer to the real and imaginary parts respectively. In the straightforward case of real valued modes, $\mathcal{S}_{i}=0$ allowing us to project out the field summation that occurs on both sides of Eq. (97). We thus derive an explicit form for the mode dispersion equation for both TM and TM polarizations

$$
1=-\frac{\omega_{L} C_{L} A_{\mathrm{WSC}} f_{L} g_{m}}{4 \pi} \ln |\delta \omega| \frac{\delta \mathcal{E}_{F}}{\mathcal{E}_{L}} .
$$

For complex valued Bloch modes, the set $\mathcal{L}$ contains a conjugate pair, i.e., $\mathcal{L}=\left\{\psi_{L}\left(\boldsymbol{k}_{L, 1}, \boldsymbol{r}\right), \psi_{L}^{*}\left(\boldsymbol{k}_{L, 1}, \boldsymbol{r}\right)\right\}$. The left-hand side of Eq. (97) then becomes

$$
\begin{aligned}
& \sum_{l \in \mathcal{L}} t_{l} \psi_{L}\left(\boldsymbol{k}_{L, 1}, \mathcal{T}_{l-1}^{T} \boldsymbol{r}\right)=\left(t_{1}+t_{2}\right) \operatorname{Re} \psi_{L}\left(\boldsymbol{k}_{L, 1}, \boldsymbol{r}\right) \\
&+i\left(t_{1}-t_{2}\right) \operatorname{Im} \psi_{L}\left(\boldsymbol{k}_{L, 1}, \boldsymbol{r}\right),
\end{aligned}
$$

while the terms $\mathcal{S}_{r}$ and $\mathcal{S}_{i}$, which occur on the right-hand side of Eq. (97), transform into

$$
\begin{aligned}
\mathcal{S}_{r} & =\frac{\delta \mathcal{E}_{R}}{\mathcal{E}_{L}}\left(t_{1}+t_{2}\right) \operatorname{Re} \psi_{L}\left(\boldsymbol{k}_{L, 1}, \boldsymbol{r}\right), \\
\mathcal{S}_{i} & =\frac{\delta \mathcal{E}_{I}}{\mathcal{E}_{L}}\left(t_{1}-t_{2}\right) \operatorname{Im} \psi_{L}\left(\boldsymbol{k}_{L, 1}, \boldsymbol{r}\right) .
\end{aligned}
$$

Thus, there are two independent solutions of Eq. (97), respectively for $t_{1}-t_{2}=0$ and $t_{1}+t_{2}=0$. When $t_{1}-t_{2}=0$, we have $\mathcal{S}_{i}=0$ and can project out the term $2 t_{1} \operatorname{Re} \psi_{L}\left(\boldsymbol{k}_{L, 1}, \boldsymbol{r}\right)$ on both sides of Eq. (97) to obtain

$$
1=-\frac{\omega_{L} C_{L} A_{\mathrm{WSC}} f_{L} g_{m_{r}}}{4 \pi} \ln |\delta \omega| \frac{\delta \mathcal{E}_{R}}{\mathcal{E}_{L}} .
$$

Correspondingly, $t_{1}+t_{2}=0$ leads to

$$
1=-\frac{\omega_{L} C_{L} A_{\mathrm{WSC}} f_{L} g_{m_{i}}}{4 \pi} \ln |\delta \omega| \frac{\delta \mathcal{E}_{I}}{\mathcal{E}_{L}} .
$$

[1] S. John, Phys. Rev. Lett. 58, 2486 (1987)

[2] E. Yablonovitch, Phys. Rev. Lett. 58, 2059 (1987).

[3] J. D. Joannopoulos, R. D. Meade, and J. N. Winn, Photonic Crystals: Molding the Flow of Light (Princeton University Press, 1995).

[4] S. Fan, M. Yanik, Z. Wang, S. Sandhu, and M. Povinelli, J. Lightwave Technol. 24, 4493 (2006).

[5] M. Notomi, A. Shinya, S. Mitsugi, G. Kira, E. Kuramochi, and T. Tanabe, Opt. Express 13, 2678 (2005).

[6] K. Busch, R. B. Wehrspohn, S. Lölkes, and H. Föll, Photonic Crystals : Advances in Design, Fabrication, and Characterization (John Wiley \& Sons, 2004).

[7] S. G. Johnson, M. Ibanescu, M. A. Skorobogatiy, O. Weisberg, J. D. Joannopoulos, and Y. Fink, Phys. Rev. E 65, 066611 (2002).

[8] M. J. Ward and J. B. Keller, SIAM J. Appl. Math. 53, 770 (1993).

[9] C. G. Poulton, L. C. Botten, R. C. McPhedran, N. A. Nicorovici, and A. B. Movchan, SIAM J. Appl. Math. 61, 1706 (2001).

[10] K. B. Dossou, R. C. McPhedran, L. C. Botten, A. A. Asatryan, and C. M. de Sterke, Opt. Express 15, 4753 (2007).

[11] R. C. McPhedran, L. C. Botten, J. McOrist, A. A. Asatryan, C. M. de Sterke, and N. A. Nicorovici, Physical
Review E 69, 016609 (pages 16) (2004).

[12] S. Wilcox, L. C. Botten, R. C. McPhedran, C. G. Poulton, and C. M. de Sterke, Phys. Rev. E 71, 056606 (pages 11) (2005).

[13] L. C. Botten, K. B. Dossou, S. Wilcox, R. C. McPhedran, C. M. de Sterke, N. A. Nicorovici, and A. A. Asatryan, Int. J. Microwave and Optical Technology 1, 133 (2006), paper IJMOT-2006-5-42.

[14] K. Dossou, M. A. Byrne, and L. C. Botten, J. Comput. Phys. 219, 120 (2006).

[15] J. M. Luttinger and W. Kohn, Phys. Rev. 97, 869 (1955).

[16] W. Kohn and J. M. Luttinger, Phys. Rev. 98, 915 (1955).

[17] P. M. Morse and H. Feshbach, Methods of theoretical physics. Part 1 (McGraw-Hill, New York, 1953).

[18] http://ab-initio.mit.edu/photons/tutorial/.

[19] E. Yablonovitch, J. Mod. Opt. 41, 173 (1994).

[20] O. Painter, J. Vuckovic, and A. Scherer, J. Opt. Soc. Am. B 16, 275 (1999).

[21] E. N. Economou, Green's functions in quantum physics, vol. 7 of Springer Series in Solid-State Sciences (Springer-Verlag, Berlin, 1983), 2nd ed.

[22] L. C. Botten, N. A. Nicorovici, R. C. McPhedran, C. M. de Sterke, and A. A. Asatryan, Phys. Rev. E 64, 046603 (2001). 